\title{
High-Energy Scatterings in Infinite-Derivative Field Theory and Ghost-Free Gravity
}

\author{
Spyridon Talaganis and Anupam Mazumdar \\ Consortium for Fundamental Physics, Lancaster University, LA1 4YB, UK
}

September 9, 2016

\begin{abstract}
In this paper, we will consider scattering diagrams in the context of infinitederivative theories. First, we examine a finite-order, higher-derivative scalar field theory and find that we cannot eliminate the growth of scattering diagrams for large external momenta. Then, we employ an infinite-derivative scalar toy model and obtain that the external momentum dependence of scattering diagrams is convergent as the external momenta become very large. In order to eliminate the external momentum growth, one has to dress the bare vertices of the scattering diagrams by considering renormalised propagator and vertex loop corrections to the bare vertices. Finally, we investigate scattering diagrams in the context of a scalar toy model which is inspired by a ghost-free and singularity-free infinite-derivative theory of gravity, where we conclude that infinite derivatives can eliminate the external momentum growth of scattering diagrams and make the scattering diagrams convergent in the ultraviolet.
\end{abstract}




\section{Contents}

1 Introduction 1

2 Scatterings in scalar field theory with higher-derivative interactions 5

2.1 Dressing the propagator . . . . . . . . . . . 6 6

2.1.1 1-loop, 3-point diagram with bare vertices and bare propagators 10

2.2 Dressing the vertices by making vertex loop corrections to the bare vertices ........................ 11

2.3 Dressing the vertices by making propagator \& vertex loop corrections to the bare vertices . . . . . . . . . . . . . . . . . 13

3 Scatterings in infinite-derivative theory 15

3.1 Dressing the propagator . . . . . . . . . . . . 17

3.2 Dressing the vertices by making vertex loop corrections to the bare vertices .......................... 18

3.3 Dressing the vertices by making propagator \& vertex loop corrections to the bare vertices . . . . . . . . . . . . . . . 20 20

4 Scattering in infinite-derivative theories of gravity 22

4.1 Dressing the propagator and the vertices . . . . . . . . . . 27 27

5 Conclusions 30

6 Acknowledgements 31

7 Appendix 31

A Definitions and Conventions in Euclidean Space 31

\section{Introduction}

Scattering diagrams play an important role in Quantum Field Theory (QFT). By studying scattering diagrams, one can obtain the scattering matrix element and, ultimately, the cross section. A cross section that blows up at high energies indicates 
an unphysical theory. Typically, in non-renormalisable theories, the cross section blows up at finite-order, see [1]. For instance, higher than 2-derivative scalar field theories are one such example. Another example is indeed General Relativity (GR); also, in supergravity, see [2], where high-energy scatterings of gravitons have been studied. Besides studying whether the amplitudes are finite or not, there are very interesting applications in cosmology and in formation of mini black holes in transPlankian scatterings of plane waves [3, 4, 5, 6, 7, 8, 9, 10, 11]. In all these cases, the cross section of a scattering diagram, especially involving gravitons, blows up for large external momenta, i.e., in the ultraviolet (UV). On the other hand, string theory has been conjectured to be UV-finite [12]; however, the problem here lies in higher-order corrections in string coupling $g_{s}$ and $\alpha^{\prime}$, which would naturally induce corrections beyond Einstein-Hilbert action. Unfortunately, many of these corrections cannot be computed so easily in a time-dependent cosmological background. Nevertheless, there has been many studies in a fixed background in the context of string scatterings, see [13, 14, 15, 16], see for details [12, 17]. Indeed, none of these analyses motivated from strings or supergravity can probe the region of space-time singularity; neither string theory nor supergravity in its current form can avoid forming a black hole or cosmological singularity. Besides string theory, there are other approaches of quantum gravity, such as in Loop Quantum Gravity (LQG) [18, 19], or in Causal Set approach [20], where it is possible to setup similar physical problems to study the behaviour at short distances and at small time scales, as well as high momentum scatterings.

One common thread in all these quantum and semiclassical approaches is the presence of non-locality, where the interactions happen in a finite region of spacetime. It has been conjectured by many that such non-local interactions may ameliorate the UV behaviour of scattering amplitudes, see [8, 9, 15, 16, 21, 22, 23, 24, 25, 26, 27, 28, [29, 30], see also Refs. [31, 32, 33, 34] for finite temperature effects of non-local field theories. It is also expected that any such realistic theory of quantum gravity should be able to resolve short-distance and small-timescale singular behaviour present in Einstein's gravity, both in static and in time-dependent backgrounds. Indeed, close to the singularity or close to super-Planckian energies, one would naturally expect higher-derivative corrections to the Einstein-Hilbert action. Such higher-derivative corrections may as well open a door for non-local interactions in a very interesting way.

Typically, higher derivatives present a problem of ghost. For instance, it is well known that a quadratic curvature gravity is renormalisable, but would contain ghosts by virtue of having four derivatives in the equation of motion. The issue of ghost persists for any finite-order, higher than 2-derivative theory for any spin. The issue of ghosts can be addressed in the context of an infinite-derivative 1 theory of gravity, see Refs. [35, 37, 38, 39, 40]. The graviton propagator is definitely modified in this

\footnotetext{
${ }^{1}$ Infinite derivatives are also present in (open) string field theory [42] and in $p$-adic strings 43 . The nonlocality of the invariant string field action was shown in [44. One would naturally expect them to be present from higher-order $\alpha^{\prime}$ corrections.
} 
case as compared to the Einstein-Hilbert action. We should point out that infinitederivative theories represent a novel approach of addressing some of the most important problems physics is facing. Among other things, the formulation of the initial value problem within the context of infinite-derivative theories remains a challenge; in Ref. [41] it was shown that, in infinite-derivative theories, there are sometimes only two pieces of initial value data per pole under the assumption that temporal Fourier transforms exist. Numerically, one requires an ansatz to solve equations of motion containing infinite derivatives, such as in the case of cosmology, see Ref. [37]. In this paper, we shall avoid these important issues by working perturbatively about a specific background in Euclidean momentum space.

In particular, in Ref. [38, the authors constructed the most general covariant construction of quadratic-order gravity with infinite derivatives around Minkowski background. Similar construction is also possible around any constant-curvature backgrounds such as in deSitter and (anti)-deSitter backgrounds [45] 2. In all these constructions [38, 45], it is possible to make the graviton propagator ghost-free, with no additional poles, other than the familiar 2 massless degrees of freedom of EinsteinHilbert action, by assuming that any modification which occurs as a result of infinite derivatives can be expressed by an entire function. An entire function as such does not introduce any pole in the complex plane. Furthermore, if the choice of an entire function is such that it falls off in the UV exponentially, while in the IR the function approaches unity in order to match the expectations of GR, then it can indeed soften the UV aspects of gravitational interactions. The fact that the propagator becomes exponentially suppressed in the UV, also leads to exponential enhancement in the vertex operator by virtue of derivative interactions. The interplay between the vertices and propagator give rise to this non-locality in gravity in the UV. Indeed, this nonlocality is responsible for some nice properties, such as the resolution of cosmological and black hole type singularities $3^{3}$.

For instance, it has been shown that for the above construction, it is possible to avoid cosmological singularity for a flat Universe Refs. [37, 38, 47, 48, 49], which yields naturally a UV modification for Starobinsky inflation [50, 51, 4. It is also possible to avoid a black hole singularity in the linearised limit; the Newtonian potential is always finite in the UV in the limit $r \rightarrow 0$, close to the source, see Ref. [38, 55, 53]. In Refs. [54, 55, 56], authors have studied the time-dependent spherical collapse of matter for such non-local gravity [38], and found that the singularity can be resolved at a linear regime. Such time-dependent results are remarkable and clearly absent in Einstein Gravity and in finite-order higher-derivative modifications of gravity, such as in 4th derivative gravity [57, 58].

\footnotetext{
${ }^{2}$ The quadratic curvature action is parity-invariant and torsion-free in both these cases 38,45 .

${ }^{3}$ In [46], one can see examples of non-local field theories which are not infinite-derivative ones; however, the approach cannot be helpful to address how to ameliorate the singularity problems at short distances and small time scales.

${ }^{4}$ In GR, for a flat Universe it is extremely hard to avoid Big Bang singularity, the null congruence always converge in a finite time [36, one requires softening of gravity in the UV in order to avoid cosmological singularity [37, 38.
} 
Furthermore, in Ref. [59], a toy model has been constructed inspired by an infinitederivative extension of quadratic order gravity. Within this framework, quantum properties have been investigated, where UV divergences originating from Feynman diagrams have been studied explicitly up to 2-loop order, and it was found that the Feynman diagrams become finite. A generic prescription was also provided on how to make higher loops finite and, in fact, renormalisable [59].

Inspired by these recent developments, the aim of this paper is to study the high energy scatterings for ghost-free and infinite-derivative theories 5 . We will study the $s, t, u$ channels of scattering diagrams for a scalar field theory. In this respect we will be extending some of earlier the computations of Refs. [30] and [59]. We will also study scattering diagrams within the scalar toy model of infinite-derivative quadratic curvature ghost-free and singularity-free gravity. In particular, we will show the following computations:

\section{2-derivative massless scalar field, with higher derivative interactions:} We will consider tree-level scattering diagrams, computed in Euclidean space. Then, we will look at the external momentum dependence of the scattering diagram if we insert a 1-loop diagram in the middle. Next, we will replace the bare propagator in the tree-level diagram with the dressed one and see how the external momentum dependence of the diagram is modified. Finally, we will consider scattering diagrams with dressed vertices and propagators. In all cases, we will find that the scattering diagrams blow up in the UV limit. We will also compute the scattering diagrams by taking into account dressed propagators and dressed vertices, and the result would be the same.

2. Infinite-derivative Lagrangian and interactions: The results of the first computation motivate us to study a ghost-free, infinite-derivative Lagrangian with interaction terms containing infinite derivatives. We will show that the scattering amplitude still blows up with and without dressed propagator. However, dressing the vertices by taking renormalised propagator and vertex loop corrections to the bare vertices eliminates the external momentum growth of the scattering amplitudes in the limit of the centre-of-mass energy going to infinity.

\section{Scalar toy model of infinite-derivative, ghost-free and singularity-free} gravity: By taking the cue from our previous computations, we will then study a scalar toy model motivated from an infinite-derivative, ghost-free and singularity-free theory of gravity [37, 38, 59]. We will show that a similar conclusion holds true for this class of action, where dressing the vertices by taking

\footnotetext{
${ }^{5}$ In principle, one can discuss breakdown of partial wave unitarity in Minkowski spacetime. A partial wave unitarity bound does not mean that beyond some energy scale unitarity is lost. It merely says that unitarity would be lost if perturbativity were assumed. In our case, we cannot define partial wave unitarity bound in Euclidean spacetime, as we shall see; instead we are keen to understand the scattering amplitudes which do not become arbitrarily large. This issue will become clear at later stages.
} 


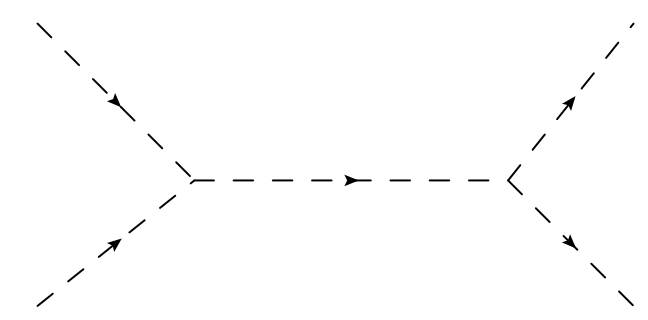

Figure 1: The $s$-channel, tree-level scattering diagram $p_{1} p_{2} \rightarrow p_{3} p_{4}$.
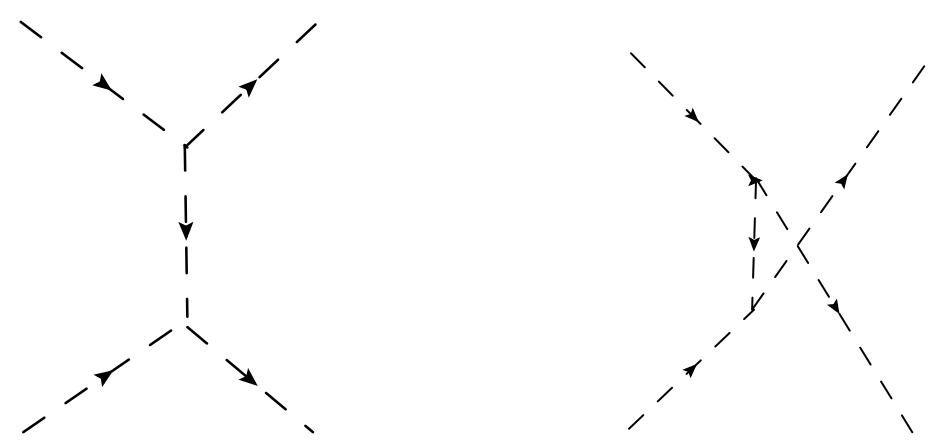

Figure 2: Left: The $t$-channel, tree-level scattering diagram $p_{1} p_{2} \rightarrow p_{3} p_{4}$. Right: The $u$-channel, tree-level scattering diagram $p_{1} p_{2} \rightarrow p_{3} p_{4}$.

both propagator and vertex loop corrections to the bare vertices makes, at sufficiently high loop order, the external momentum dependence of any scattering diagram convergent in the UV.

The paper is organised as follows: in section 2, we introduce a finite-order higherderivative scalar field theory and examine the UV external momentum dependence of scattering diagrams. In section 3, we write down an infinite-derivative scalar field theory and study the external momentum dependence of scattering diagrams. In section 4, we investigate external momentum dependence of scatterings of a scalar field theory analogue of infinite-derivative theory of gravity, and in section 5, we conclude by summarising our results.

\section{Scatterings in scalar field theory with higher- derivative interactions}

Let us now begin with a simple massless scalar field with a higher-derivative interaction term:

$$
S=S_{\text {free }}+S_{\text {int }}
$$

where

$$
S_{\text {free }}=\frac{1}{2} \int d^{4} x(\phi \square \phi)
$$


and

$$
S_{\text {int }}=\lambda \int d^{4} x(\phi \square \phi \square \phi),
$$

where we treat $\lambda \ll \mathcal{O}(1)$, so that we are within the perturbative limit. We will be working in an Euclidean space 6 , the propagator in the momentum space is then given by

$$
\Pi\left(k^{2}\right)=\frac{-i}{k^{2}},
$$

while the vertex factor is given by:

$$
\lambda V\left(k_{1}, k_{2}, k_{3}\right)=2 i \lambda\left(k_{1}^{2} k_{2}^{2}+k_{2}^{2} k_{3}^{2}+k_{3}^{2} k_{1}^{2}\right),
$$

where

$$
k_{1}+k_{2}+k_{3}=0 \text {. }
$$

We can compute the tree-level amplitudes for the $s, t, u$ channels, see Fig. 1 ,

$$
i \mathcal{T}_{\text {tree-level }}^{\text {s-channel }}=-\frac{25}{4} \lambda^{2} s^{4}\left(\frac{i}{s}\right)
$$

where $s=-\left(p_{1}+p_{2}\right)^{2}$. Similarly, see Fig. 2 (left),

$$
i \mathcal{T}_{\text {tree-level }}^{\text {t-channel }}=-4 \lambda^{2} s^{2}\left(t+\frac{s}{4}\right)^{2}\left(\frac{i}{t}\right)
$$

and, see Fig. 2 (right),

$$
i \mathcal{T}_{\text {tree-level }}^{\mathrm{u}-\text { channel }}=-4 \lambda^{2} s^{2}\left(u+\frac{s}{4}\right)^{2}\left(\frac{i}{u}\right)
$$

where $t=-\left(p_{1}-p_{3}\right)^{2}$ and $u=-\left(p_{1}-p_{4}\right)^{2}$. Hence, the total amplitude is given by:

$$
\mathcal{T}_{\text {tree-level }}=-4 \lambda^{2} s^{2}\left(\frac{\left(\frac{5 s}{4}\right)^{2}}{s}+\frac{\left(t+\frac{s}{4}\right)^{2}}{t}+\frac{\left(u+\frac{s}{4}\right)^{2}}{u}\right) .
$$

Since the scattering matrix element $\mathcal{T}_{\text {tree-level }}$ in Eq. (2.10) blows up as $s \rightarrow-\infty$, the total cross section $\sigma_{\text {tree-level }}$ in the centre-of-mass (CM) frame (see Eqs. (A.1) \& (A.4) in appendix $\mathrm{A}$ for the definition of $\sigma$ ) also blows up as $s \rightarrow-\infty$.

\subsection{Dressing the propagator}

Since the tree-level amplitude blows up, we should now study the 1-loop, 2-point function in the propagator for the above interaction, see Eq. (2.1). We can compute the

\footnotetext{
${ }^{6}$ In Minkowski space ("mostly plus" metric signature), $k^{2}=-k_{0}^{2}+\vec{k}^{2}$, where $\vec{k}^{2}=k_{1}^{2}+k_{2}^{2}+k_{3}^{2}$. After analytic continuation, $k_{E}^{2}=k_{4}^{2}+\vec{k}^{2}$, where $k_{4}=-i k_{0}$. For brevity, we will suppress the subscript $E$ in the notations. For the rest of the paper we will continue our computations in Euclidean space.
} 


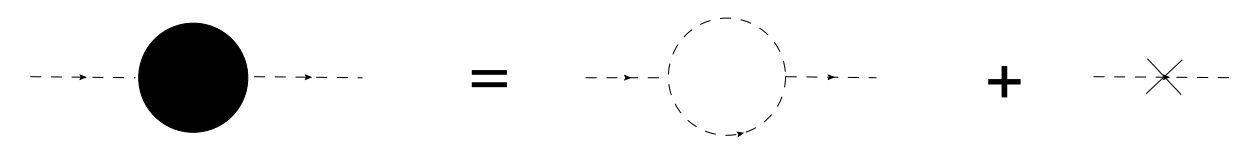

Figure 3: The 1-loop, 2-point contribution of 1PI diagrams. The cross denotes a counterterm vertex.

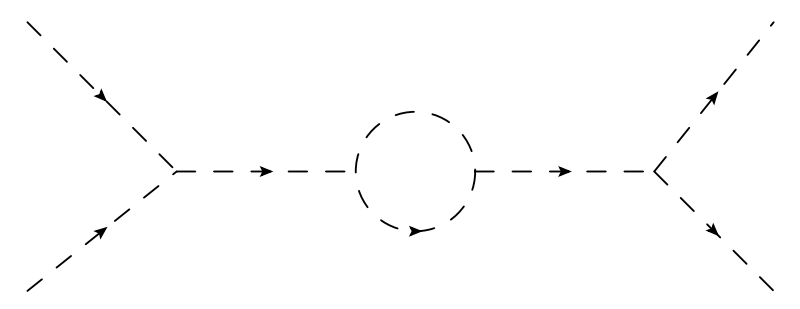

Figure 4: The $s$-channel, 1-loop scattering diagram $p_{1} p_{2} \rightarrow p_{3} p_{4}$.

1-loop, 2-point function with arbitrary external momentum, $p$. Therefore, regarding the 1-loop, 2-point function with external momenta $p,-p$ and symmetrical routing of momenta, see Fig. 3 , we have

$$
\begin{aligned}
\Gamma_{2,1}\left(p^{2}\right) & =\frac{i \lambda^{2}}{2} \int^{\Lambda} \frac{d^{4} k}{(2 \pi)^{4}} \frac{4\left[p^{2}\left(\frac{p}{2}-k\right)^{2}+p^{2}\left(\frac{p}{2}+k\right)^{2}+\left(\frac{p}{2}+k\right)^{2}\left(\frac{p}{2}-k\right)^{2}\right]^{2}}{\left(\frac{p}{2}-k\right)^{2}\left(\frac{p}{2}+k\right)^{2}} \\
& =\frac{i \lambda^{2}}{2} \int_{0}^{\Lambda} d \mathbf{k} \int_{-1}^{1} d x \frac{4 \pi \mathbf{k}^{3} \sqrt{1-x^{2}}}{(2 \pi)^{4}} \frac{4\left[p^{2}\left(\frac{p}{2}-k\right)^{2}+p^{2}\left(\frac{p}{2}+k\right)^{2}+\left(\frac{p}{2}+k\right)^{2}\left(\frac{p}{2}-k\right)^{2}\right]^{2}}{\left(\frac{p}{2}-k\right)^{2}\left(\frac{p}{2}+k\right)^{2}} \\
& =i \lambda^{2}\left(-\frac{p^{8}}{48 \pi^{2}}+\frac{\Lambda^{2} p^{6}}{8 \pi^{2}}+\frac{81 \Lambda^{4} p^{4}}{256 \pi^{2}}+\frac{17 \Lambda^{6} p^{2}}{96 \pi^{2}}+\frac{\Lambda^{8}}{32 \pi^{2}}\right)
\end{aligned}
$$

where $k$ is the internal loop momentum, $x$ is the cosine of the angle between $p$ and $k$ $(p \cdot k=\mathbf{p} \mathbf{k} x$, where $\mathbf{p}$ and $\mathbf{k}$ are the norms of $p$ and $k$ in Euclidean space) and $\Lambda$ is a hard cutoff. The counter-term, which is needed to cancel the divergences denoted by powers of $\Lambda$ in Eq. (2.11), and which should be added to the action in Eq. (2.1), is given by

$$
S_{\mathrm{ct}}=\frac{\lambda^{2} \Lambda^{2}}{16 \pi^{2}} \int d^{4} x\left(\phi \square^{3} \phi-\frac{81 \Lambda^{2}}{32} \phi \square^{2} \phi+\frac{17 \Lambda^{4}}{12} \phi \square \phi-\frac{\Lambda^{6}}{4} \phi^{2}\right),
$$

which yields

$$
\Gamma_{2,1, \mathrm{ct}}\left(p^{2}\right)=-\frac{i \lambda^{2} \Lambda^{2}}{8 \pi^{2}}\left(p^{6}+\frac{81 \Lambda^{2} p^{4}}{32}+\frac{17 \Lambda^{4} p^{2}}{12}+\frac{\Lambda^{6}}{4}\right)
$$

Thus, the renormalised 1-loop, 2-point function is

$$
\Gamma_{2,1 \mathrm{r}}\left(p^{2}\right)=\Gamma_{2,1}\left(p^{2}\right)+\Gamma_{2,1, \mathrm{ct}}\left(p^{2}\right)=-\frac{i \lambda^{2} p^{8}}{48 \pi^{2}}
$$

We observe that the maximum power of $p$ appearing in the renormalised 1-loop, 2point function with arbitrary external momenta, Eq. (2.14), is $p^{8}$. Hence, in the UV, 


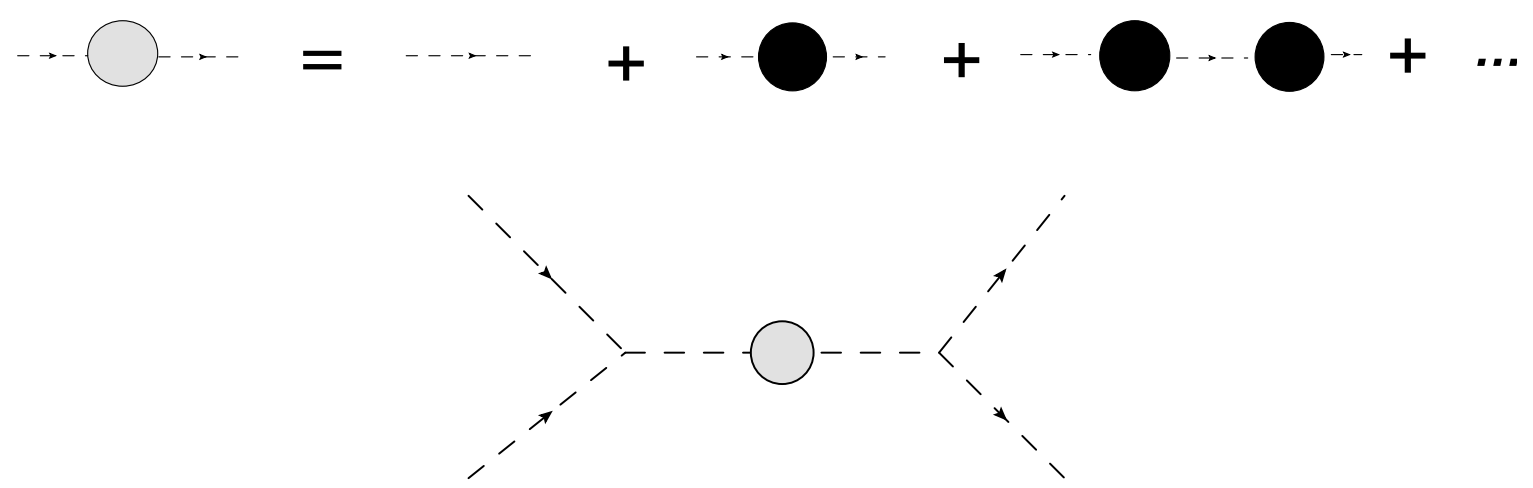

Figure 5: Top: The dressed propagator as the sum of an infinite geometric series. The dressed propagator is denoted by the shaded blob. Bottom: The $s$-channel, scattering diagram $p_{1} p_{2} \rightarrow p_{3} p_{4}$ in which the bare propagator is replaced by the dressed propagator. The shaded blob indicates a dressed propagator.

i.e., in the limit $s \rightarrow-\infty, \Gamma_{2,1 \mathrm{r}}(-s) \propto\left(p_{1}+p_{2}\right)^{8}=s^{4}$, where $\Gamma_{2,1 \mathrm{r}}$ is the renormalised 1-loop, 2-point function. Since

$$
\begin{aligned}
i \mathcal{T}_{1-\text { loop }} & =\lambda^{2}\left(p_{1}, p_{2},-p_{1}-p_{2}\right) V\left(-p_{3},-p_{4}, p_{1}+p_{2}\right)\left(\frac{i}{s}\right)^{2} \Gamma_{2,1 \mathrm{r}}(-s) \\
& +\lambda^{2} V\left(p_{1},-p_{3}, p_{3}-p_{1}\right) V\left(p_{2},-p_{4}, p_{1}-p_{3}\right)\left(\frac{i}{t}\right)^{2} \Gamma_{2,1 \mathrm{r}}(-t) \\
& +\lambda^{2} V\left(p_{1},-p_{4}, p_{4}-p_{1}\right) V\left(p_{2},-p_{3}, p_{1}-p_{4}\right)\left(\frac{i}{u}\right)^{2} \Gamma_{2,1 \mathrm{r}}(-u)
\end{aligned}
$$

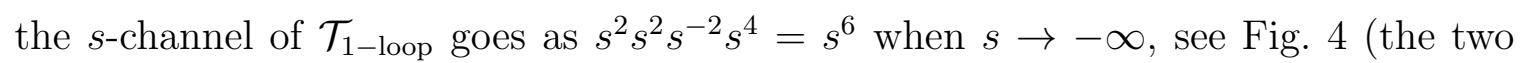
bare propagators go as $1 / s$ each while the two bare vertices go as $s^{2}$ each). Hence, as $s \rightarrow-\infty, \mathcal{T}_{1-\text { loop }}^{\text {s-chanel }}$ diverges. $\mathcal{T}_{1-\text { loop }}^{\text {t-channel }}$ and $\mathcal{T}_{1-\text { loop }}^{\mathrm{u} \text {-chanel }}$ also diverge except for $\theta=0$ and $\theta=\pi$, respectively.

Now what if we had an infinite series of loops in the scattering diagrams, see Fig. 5 (top), that is, if we had replaced the bare propagator with the dressed propagator? As we shall see below, the external momentum dependence of the 1-loop, 2-point function shall actually determine the UV behaviour of the dressed propagator.

The dressed propagator, see Fig. 5 (top), represents the geometric series of all the graphs with 1-loop, 2-point insertions, analytically continued to the entire complex $p^{2}$-plane. Mathematically, the dressed propagator, $\widetilde{\Pi}\left(p^{2}\right)$, is given by [59]

$$
\widetilde{\Pi}\left(p^{2}\right)=\frac{\Pi\left(p^{2}\right)}{1-\Pi\left(p^{2}\right) \Gamma_{2,1 \mathrm{r}}\left(p^{2}\right)} .
$$


Hence, for our example, we have

$$
\begin{aligned}
\widetilde{\Pi}\left(p^{2}\right) & =\frac{-\frac{i}{p^{2}}}{1-\left(-\frac{i}{p^{2}}\right)\left(-\frac{i \lambda^{2} p^{8}}{48 \pi^{2}}\right)} \\
& =\frac{-i}{p^{2}+\frac{\lambda^{2} p^{8}}{48 \pi^{2}}} .
\end{aligned}
$$

For large $p, p^{8}$ dominates $p^{2}$ in the denominator of Eq. (2.17), and we have

$$
\widetilde{\Pi}\left(p^{2}\right) \approx-\frac{48 \pi^{2} i}{\lambda^{2} p^{8}}
$$

Since

$$
\begin{aligned}
i \mathcal{T}_{\text {dressed }} & =\lambda^{2} V\left(p_{1}, p_{2},-p_{1}-p_{2}\right) V\left(-p_{3},-p_{4}, p_{1}+p_{2}\right) \widetilde{\Pi}(-s) \\
& +\lambda^{2} V\left(p_{1},-p_{3}, p_{3}-p_{1}\right) V\left(p_{2},-p_{4}, p_{1}-p_{3}\right) \widetilde{\Pi}(-t) \\
& +\lambda^{2} V\left(p_{1},-p_{4}, p_{4}-p_{1}\right) V\left(p_{2},-p_{3}, p_{1}-p_{4}\right) \widetilde{\Pi}(-u)
\end{aligned}
$$

then, if we replace the bare propagator with the dressed propagator in the tree-level scattering diagrams, see Fig. 5 (bottom), we will have

$$
\begin{aligned}
& \mathcal{T}_{\text {dressed }}^{\text {S-channel }}=-\frac{25}{4} \lambda^{2} \frac{s^{3}}{1-\frac{\lambda^{2} s^{3}}{48 \pi^{2}}}, \\
& \mathcal{T}_{\text {dressed }}^{\text {t-channel }}=-4 \lambda^{2}\left(\frac{3 s}{4}-\frac{s}{2} \cos \theta\right)^{2} \frac{2 s}{(1-\cos \theta)\left[1-\frac{\lambda^{2} s^{3}(1-\cos \theta)^{3}}{384 \pi^{2}}\right]}, \\
& \mathcal{T}_{\text {dressed }}^{\mathrm{u}-\text { channel }}=-4 \lambda^{2}\left(\frac{3 s}{4}+\frac{s}{2} \cos \theta\right)^{2} \frac{2 s}{(1+\cos \theta)\left[1-\frac{\lambda^{2} s^{3}(1+\cos \theta)^{3}}{384 \pi^{2}}\right]} .
\end{aligned}
$$

Hence, we can make the following observations:

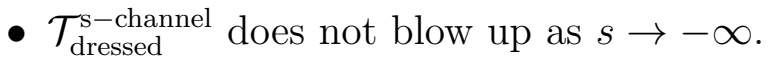

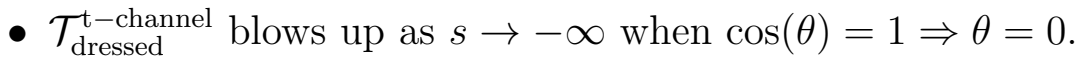

- Similarly, $\mathcal{T}_{\text {dressed }}^{\mathrm{u}-\text { channel }}$ blows up as $s \rightarrow-\infty$ when $\cos (\theta)=-1 \Rightarrow \theta=\pi$.

Since we have that $\mathcal{T}_{\text {dressed }}=\mathcal{T}_{\text {dressed }}^{\mathrm{s}-\text { chanel }}+\mathcal{T}_{\text {dressed }}^{\mathrm{t} \text {-chanel }}+\mathcal{T}_{\text {dressed }}^{\mathrm{u} \text {-channel }}$, one can verify that the total cross section $\sigma_{\text {dressed }}$ corresponding to $\mathcal{T}_{\text {dressed }}$ blows up as $s \rightarrow-\infty$. The summary is that the dressed propagator is not sufficient to prevent the scattering diagram from blowing up as $s \rightarrow-\infty$ since the polynomial suppression coming from the dressed propagator cannot overcome the polynomial enhancement originating from the two bare vertices in Fig. 5 (bottom). In subsection 2.2, we shall dress the vertices to see whether we can eliminate the external momentum divergences of the scattering diagrams. 


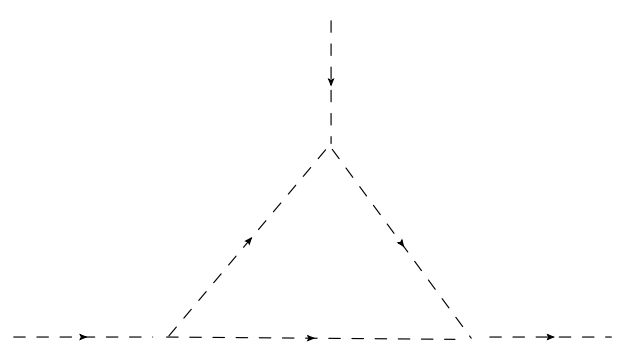

Figure 6: 1-loop, 3-point diagram with bare vertices and bare internal propagators and symmetrical routing of momenta. The external momenta are $p_{1}, p_{2}, p_{3}$ and the internal (that is, inside the loop) momenta are $k+\frac{p_{1}}{3}-\frac{p_{2}}{3}, k+\frac{p_{2}}{3}-\frac{p_{3}}{3}, k+\frac{p_{3}}{3}-\frac{p_{1}}{3}$.

\subsubsection{1-loop, 3-point diagram with bare vertices and bare propagators}

As a prelude to subsection 2.2, suppose we consider a 1-loop, 3-point diagram, see Fig. 6, with external momenta $p_{1}, p_{2}$ and $p_{3}$ (we assume that the propagators and the vertices are bare), and symmetrical routing of momenta. Then the propagators in the 1-loop triangle are given by Eq. (2.4):

$$
-i\left(k+\frac{p_{1}}{3}-\frac{p_{2}}{3}\right)^{-2},-i\left(k+\frac{p_{2}}{3}-\frac{p_{3}}{3}\right)^{-2},-i\left(k+\frac{p_{3}}{3}-\frac{p_{1}}{3}\right)^{-2},
$$

and the vertex factors are given by Eq. (2.5):

$$
\begin{aligned}
& 2 i \lambda\left(p_{2}^{2}\left(k+\frac{p_{1}}{3}-\frac{p_{2}}{3}\right)^{2}+p_{2}^{2}\left(k+\frac{p_{2}}{3}-\frac{p_{3}}{3}\right)^{2}+\left(k+\frac{p_{1}}{3}-\frac{p_{2}}{3}\right)^{2}\left(k+\frac{p_{2}}{3}-\frac{p_{3}}{3}\right)^{2}\right), \\
& 2 i \lambda\left(p_{3}^{2}\left(k+\frac{p_{2}}{3}-\frac{p_{3}}{3}\right)^{2}+p_{3}^{2}\left(k+\frac{p_{3}}{3}-\frac{p_{1}}{3}\right)^{2}+\left(k+\frac{p_{2}}{3}-\frac{p_{3}}{3}\right)^{2}\left(k+\frac{p_{3}}{3}-\frac{p_{1}}{3}\right)^{2}\right), \\
& 2 i \lambda\left(p_{1}^{2}\left(k+\frac{p_{3}}{3}-\frac{p_{1}}{3}\right)^{2}+p_{1}^{2}\left(k+\frac{p_{1}}{3}-\frac{p_{2}}{3}\right)^{2}+\left(k+\frac{p_{3}}{3}-\frac{p_{1}}{3}\right)^{2}\left(k+\frac{p_{1}}{3}-\frac{p_{2}}{3}\right)^{2}\right) .
\end{aligned}
$$

Hence, the 1-loop, 3-point diagram, $\Gamma_{3,1}\left(p^{2}\right)$, will be given by

$$
\begin{aligned}
\Gamma_{3,1}\left(p^{2}\right) & =i \lambda^{3} \int^{\Lambda} \frac{d^{4} k}{(2 \pi)^{4}}\left[\frac{8\left(p_{2}^{2}\left(k+\frac{p_{1}}{3}-\frac{p_{2}}{3}\right)^{2}+p_{2}^{2}\left(k+\frac{p_{2}}{3}-\frac{p_{3}}{3}\right)^{2}+\left(k+\frac{p_{1}}{3}-\frac{p_{2}}{3}\right)^{2}\left(k+\frac{p_{2}}{3}-\frac{p_{3}}{3}\right)^{2}\right)}{\left(k+\frac{p_{1}}{3}-\frac{p_{2}}{3}\right)^{2}\left(k+\frac{p_{2}}{3}-\frac{p_{3}}{3}\right)^{2}\left(k+\frac{p_{3}}{3}-\frac{p_{1}}{3}\right)^{2}}\right. \\
& \times\left(p_{3}^{2}\left(k+\frac{p_{2}}{3}-\frac{p_{3}}{3}\right)^{2}+p_{3}^{2}\left(k+\frac{p_{3}}{3}-\frac{p_{1}}{3}\right)^{2}+\left(k+\frac{p_{2}}{3}-\frac{p_{3}}{3}\right)^{2}\left(k+\frac{p_{3}}{3}-\frac{p_{1}}{3}\right)^{2}\right) \\
& \left.\times\left(p_{1}^{2}\left(k+\frac{p_{3}}{3}-\frac{p_{1}}{3}\right)^{2}+p_{1}^{2}\left(k+\frac{p_{1}}{3}-\frac{p_{2}}{3}\right)^{2}+\left(k+\frac{p_{3}}{3}-\frac{p_{1}}{3}\right)^{2}\left(k+\frac{p_{1}}{3}-\frac{p_{2}}{3}\right)^{2}\right)\right] .
\end{aligned}
$$

After integration with respect to the internal loop momentum $k$ and renormalisation of the loop integral divergences, i.e., the terms involving powers of $\Lambda$, we are left with a polynomial function of the three external momenta $p_{1}, p_{2}, p_{3}$. We will require these computations in the following subsection. 


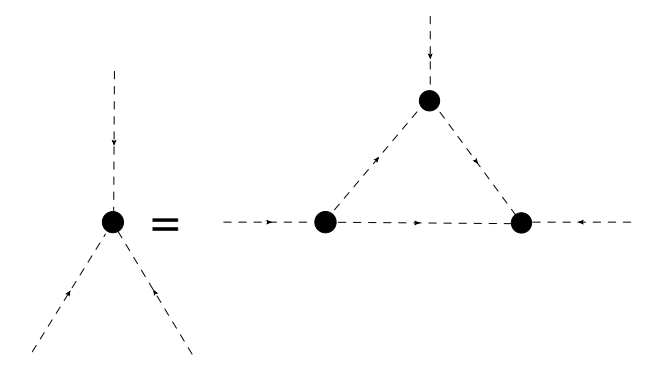

Figure 7: 3-point diagram constructed out of lower-loop 2-point \& 3-point diagrams. The dark blobs indicate renormalised vertex corrections and the dashed lines inside the triangle denote bare internal propagators. The loop order of the dark blob on the left is $n$ while the loop order of the dark blobs on the right is $n-1$. The external momenta are $p_{1}, p_{2}, p_{3}$ and the internal (that is, inside the loop) momenta are $k+\frac{p_{1}}{3}-\frac{p_{2}}{3}, k+\frac{p_{2}}{3}-\frac{p_{3}}{3}, k+\frac{p_{3}}{3}-\frac{p_{1}}{3}$.

\subsection{Dressing the vertices by making vertex loop corrections to the bare vertices}

Based on the results of subsection 2.1.1, suppose we want to dress the vertices by making renormalised vertex loop corrections to the bare vertices at the left- and rightends of the scattering diagrams, see Fig. 7. As we saw in Eq. 2.25), both the bare propagators and the bare vertices can be written in terms of powers of momenta. After integration with respect to the internal loop momentum $k$, we obtain a polynomial expression involving powers of the external momenta $p_{1}, p_{2}, p_{3}$. As the loop-order increases, the 3-point function can still be written as a polynomial function of the external momenta; this happens because, as previously, the (bare) propagators are polynomials in momenta while the (dressed) vertices are also polynomials in momenta. Therefore, we expect the external momentum dependence of the 3-point function, see Fig 7, in the UV limit, i.e., as $p_{i} \rightarrow \infty$, where $i=1,2,3$, in terms of the three external momenta, $p_{1}, p_{2}, p_{3}$, to follow as:

$$
\Gamma_{3} \stackrel{U V}{\longrightarrow} \sum_{\alpha, \beta, \gamma} p_{1}^{2 \alpha} p_{2}^{2 \beta} p_{3}^{2 \gamma}
$$

with the convention

$$
\alpha \geq \beta \geq \gamma
$$

The reason we expect the external momentum dependence of 3-point function to be given by Eq. (2.26) is that, once all the (lower-) loop subdiagrams have been integrated out, what remains are polynomial expressions in terms of the corresponding external momenta. Some of these external momenta can then become the internal loop momentum in a subsequent higher-loop diagram.

First, let us consider how one can get the largest sum of all the exponents, i.e., $\alpha+\beta+\gamma$. Although all the arguments below can be conducted for three different sets of exponents in the three 3-point vertices making up the 1-loop triangle, see Fig. 7 , for simplicity, here we will look at what happens when all the three vertices have the same exponents. 
Clearly, the best way to obtain the largest exponents for the external momenta is to have the $\alpha$ exponent correspond to the external momenta. Assuming a symmetric distribution of $(\beta, \gamma)$ among the internal loops and considering the $n$-loop, 3-point diagram with symmetrical routing of momenta, see Fig. 7 , the propagators in the 1-loop triangle are given by Eq. (2.23) and the vertex factors are ${ }^{7}$

$$
\begin{aligned}
& i p_{1}^{2 \alpha^{n-1}}\left(k+\frac{p_{3}}{3}-\frac{p_{1}}{3}\right)^{2 \beta^{n-1}}\left(k+\frac{p_{1}}{3}-\frac{p_{2}}{3}\right)^{2 \gamma^{n-1}}, \\
& i p_{2}^{2 \alpha^{n-1}}\left(k+\frac{p_{1}}{3}-\frac{p_{2}}{3}\right)^{2 \beta^{n-1}}\left(k+\frac{p_{2}}{3}-\frac{p_{3}}{3}\right)^{2 \gamma^{n-1}}, \\
& i p_{3}^{2 \alpha^{n-1}}\left(k+\frac{p_{2}}{3}-\frac{p_{3}}{3}\right)^{2 \beta^{n-1}}\left(k+\frac{p_{3}}{3}-\frac{p_{1}}{3}\right)^{2 \gamma^{n-1}} .
\end{aligned}
$$

Conservation of momenta then yields, in the UV, i.e., as $p_{i} \rightarrow \infty$, where $i=1,2,3$,

$$
\begin{aligned}
\Gamma_{3, n} & \longrightarrow \int \frac{\mathrm{d}^{4} k}{(2 \pi)^{4}}\left[\frac{p_{1}^{2 \alpha^{n-1}} p_{2}^{2 \alpha^{n-1}} p_{3}^{2 \alpha^{n-1}}}{\left(k+\frac{p_{1}}{3}-\frac{p_{2}}{3}\right)^{2}\left(k+\frac{p_{2}}{3}-\frac{p_{3}}{3}\right)^{2}\left(k+\frac{p_{3}}{3}-\frac{p_{1}}{3}\right)^{2}}\right. \\
& \left.\times\left(k+\frac{p_{1}}{3}-\frac{p_{2}}{3}\right)^{2\left(\beta^{n-1}+\gamma^{n-1}\right)}\left(k+\frac{p_{2}}{3}-\frac{p_{3}}{3}\right)^{2\left(\beta^{n-1}+\gamma^{n-1}\right)}\left(k+\frac{p_{3}}{3}-\frac{p_{1}}{3}\right)^{2\left(\beta^{n-1}+\gamma^{n-1}\right)}\right],
\end{aligned}
$$

where $p_{1}, p_{2}, p_{3}$ are the external momenta for the 1-loop triangle and the superscript in the $\alpha, \beta, \gamma$ indicates that these are coefficients that one obtains from contributions up to $n-1$ loop level. Now, let us proceed to obtain the $n$-th loop coefficients. We can read from Eq. 2.29):

$$
\alpha^{n}=\beta^{n}=\gamma^{n}=\alpha^{n-1}+2\left(\beta^{n-1}+\gamma^{n-1}\right) .
$$

For 3-point bare vertices, we have now $\alpha^{0}=\beta^{0}=1$ and $\gamma^{0}=0$. As $n$ increases, $\alpha^{n}, \beta^{n}$ and $\gamma^{n}$ increase; this means that, as the number of loops increases, the external momentum dependences of the dressed vertices become larger and larger as the external momenta become larger.

If we now dress the vertices by making renormalised vertex loop corrections to the bare vertices at the left- and right-ends of the tree-level scattering diagrams, we will have, see Fig. 9 (for $n \geq 1, \alpha^{n}=\beta^{n}=\gamma^{n}$ ),

$$
\begin{aligned}
& \mathcal{T}_{\text {vertex corrections }}^{\text {s-channel }} \sim s^{2 \alpha^{n}}\left(\frac{s}{2}\right)^{4 \alpha^{n}} \frac{1}{s}, \\
& \mathcal{T}_{\text {vertex corrections }}^{\mathrm{t}-\text { channel }} \sim t^{2 \alpha^{n}}\left(\frac{s}{2}\right)^{4 \alpha^{n}} \frac{1}{t}=\left[\frac{s}{2}(1-\cos \theta)\right]^{2 \alpha^{n}-1}\left(\frac{s}{2}\right)^{4 \alpha^{n}}, \\
& \mathcal{T}_{\text {vertex corrections }}^{\mathrm{u}-\text { channel }} \sim u^{2 \alpha^{n}}\left(\frac{s}{2}\right)^{4 \alpha^{n}} \frac{1}{u}=\left[\frac{s}{2}(1+\cos \theta)\right]^{2 \alpha^{n}-1}\left(\frac{s}{2}\right)^{4 \alpha^{n}} .
\end{aligned}
$$

Since $\alpha^{0}=\beta^{0}=1$ and $\gamma^{0}=0$, using Eq. (2.30), we can see that $\alpha^{1}=3$; therefore, $\alpha^{n} \geq 3$ for $n \geq 1$. Hence, we can make the following observations from the above expressions:

\footnotetext{
${ }^{7}$ The superscripts in $\alpha^{n-1}, \beta^{n-1}, \gamma^{n}-1$ denote the loop-order; clearly, they are not powers.
} 


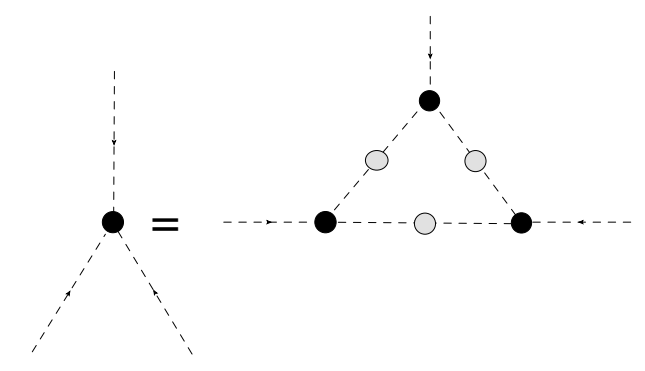

Figure 8: 3-point diagram constructed out of lower-loop 2-point \& 3-point diagrams. The shaded blobs indicate dressed internal propagators and the dark blobs indicate renormalised vertex corrections. The loop order of the dark blob on the left is $n$ while the loop order of the dark blobs on the right is $n-1$. The external momenta are $p_{1}, p_{2}, p_{3}$ and the internal (that is, inside the loop) momenta are $k+\frac{p_{1}}{3}-\frac{p_{2}}{3}, k+\frac{p_{2}}{3}-\frac{p_{3}}{3}, k+\frac{p_{3}}{3}-\frac{p_{1}}{3}$.

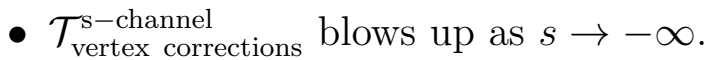

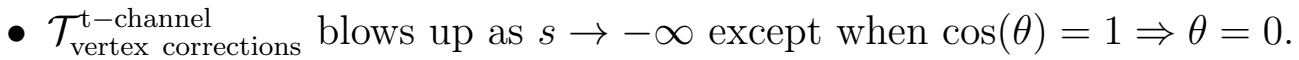

- Similarly, $\mathcal{T}_{\text {vertex corrections }}^{\mathrm{u}-\text { bhannel }}$ blows up as $s \rightarrow-\infty$ except when $\cos (\theta)=-1 \Rightarrow$ $\theta=\pi$.

Thus, one can check that the cross section $\sigma_{\text {dressed vertices }}$ corresponding to $\mathcal{T}_{\text {vertex corrections }}=$

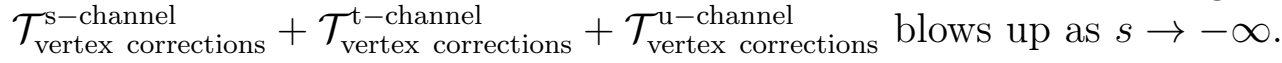

We see that dressing the vertices by making just vertex loop corrections to the bare vertices does not ameliorate the external momentum growth of scattering diagrams in the UV in our example, see Eq. (2.1). In fact, it makes the growth increase. In the next subsection, we shall dress the vertices by making both propagator and vertex loop corrections to the bare vertices at the left- and right-ends of the scattering diagrams.

\subsection{Dressing the vertices by making propagator \& vertex loop corrections to the bare vertices}

In this subsection, we shall dress the vertices by making renormalised propagator and vertex loop corrections to the bare vertices at the left- and right-ends of the scattering diagrams, see Fig. 8. Again, we expect the external momentum dependence of the 3 -point function to be given in the UV limit, i.e., as $p_{i} \rightarrow \infty$, where $i=1,2,3$, by Eq. (2.26).

As previously, the best way to obtain the largest exponents for the external momenta is to have the $\alpha$ exponent correspond to the external momenta. Assuming a symmetric distribution of $(\beta, \gamma)$ among the internal loops and considering the $n$ loop, 3-point diagram with symmetrical routing of momenta, see Fig. 8 , the (dressed) 


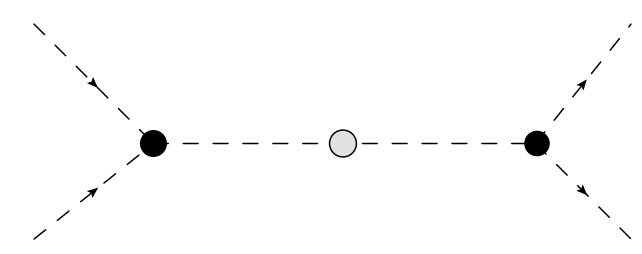

Figure 9: An $s$-channel scattering diagram $p_{1} p_{2} \rightarrow p_{3} p_{4}$. The shaded blob indicates a dressed propagator and the dark blobs indicate renormalised vertex corrections.

propagators in the 1-loop triangle are

$$
-i\left(k+\frac{p_{1}}{3}-\frac{p_{2}}{3}\right)^{-8},-i\left(k+\frac{p_{2}}{3}-\frac{p_{3}}{3}\right)^{-8},-i\left(k+\frac{p_{3}}{3}-\frac{p_{1}}{3}\right)^{-8},
$$

while the vertex factors are

$$
\begin{aligned}
& i p_{1}^{2 \alpha^{n-1}}\left(k+\frac{p_{3}}{3}-\frac{p_{1}}{3}\right)^{2 \beta^{n-1}}\left(k+\frac{p_{1}}{3}-\frac{p_{2}}{3}\right)^{2 \gamma^{n-1}}, \\
& i p_{2}^{2 \alpha^{n-1}}\left(k+\frac{p_{1}}{3}-\frac{p_{2}}{3}\right)^{2 \beta^{n-1}}\left(k+\frac{p_{2}}{3}-\frac{p_{3}}{3}\right)^{2 \gamma^{n-1}}, \\
& i p_{3}^{2 \alpha^{n-1}}\left(k+\frac{p_{2}}{3}-\frac{p_{3}}{3}\right)^{2 \beta^{n-1}}\left(k+\frac{p_{3}}{3}-\frac{p_{1}}{3}\right)^{2 \gamma^{n-1}} .
\end{aligned}
$$

Conservation of momenta then yields, in the UV, i.e., as $p_{i} \rightarrow \infty$, where $i=1,2,3$,

$$
\begin{aligned}
\Gamma_{3, n} & \longrightarrow \int \frac{\mathrm{d}^{4} k}{(2 \pi)^{4}}\left[\frac{p_{1}^{2 \alpha^{n-1}} p_{2}^{2 \alpha^{n-1}} p_{3}^{2 \alpha^{n-1}}}{\left(k+\frac{p_{1}}{3}-\frac{p_{2}}{3}\right)^{8}\left(k+\frac{p_{2}}{3}-\frac{p_{3}}{3}\right)^{8}\left(k+\frac{p_{3}}{3}-\frac{p_{1}}{3}\right)^{8}}\right. \\
& \left.\times\left(k+\frac{p_{1}}{3}-\frac{p_{2}}{3}\right)^{2\left(\beta^{n-1}+\gamma^{n-1}\right)}\left(k+\frac{p_{2}}{3}-\frac{p_{3}}{3}\right)^{2\left(\beta^{n-1}+\gamma^{n-1}\right)}\left(k+\frac{p_{3}}{3}-\frac{p_{1}}{3}\right)^{2\left(\beta^{n-1}+\gamma^{n-1}\right)}\right],
\end{aligned}
$$

where $p_{1}, p_{2}, p_{3}$ are the external momenta for the 1-loop triangle and the superscript in the $\alpha, \beta, \gamma$ indicates that these are coefficients that one obtains from contributions up to $n-1$ loop level. Now, let us proceed to obtain the $n$-th loop coefficients by inspecting Eq. (2.36), we have

$$
\alpha^{n}=\beta^{n}=\gamma^{n}=\alpha^{n-1}+2\left(\beta^{n-1}+\gamma^{n-1}\right) .
$$

For the 3-point bare vertices, we have that $\alpha^{0}=\beta^{0}=1$ and $\gamma^{0}=0$. As $n$ increases, $\alpha^{n}, \beta^{n}$ and $\gamma^{n}$ increase; this means that, as the number of loops increases, the external momentum growth of the dressed vertices increases.

If we now dress the vertices by making renormalised propagator and vertex loop corrections to the bare vertices at the left- and right-ends of the tree-level scattering 
diagrams, see Fig. 9, we obtain, as $s \rightarrow-\infty$,

$$
\begin{aligned}
& \mathcal{T}_{\text {both corrections }}^{\text {s-channel }} \sim s^{2 \alpha^{n}}\left(\frac{s}{2}\right)^{4 \alpha^{n}} \frac{1}{s^{4}}, \\
& \mathcal{T}_{\text {both corrections }}^{\text {t-channel }} \sim t^{2 \alpha^{n}}\left(\frac{s}{2}\right)^{4 \alpha^{n}} \frac{1}{t^{4}}=\left[\frac{s}{2}(1-\cos \theta)\right]^{2 \alpha^{n}-4}\left(\frac{s}{2}\right)^{4 \alpha^{n}}, \\
& \mathcal{T}_{\text {both corrections }}^{\text {u-channel }} \sim u^{2 \alpha^{n}}\left(\frac{s}{2}\right)^{4 \alpha^{n}} \frac{1}{u^{4}}=\left[\frac{s}{2}(1+\cos \theta)\right]^{2 \alpha^{n}-4}\left(\frac{s}{2}\right)^{4 \alpha^{n}} .
\end{aligned}
$$

Since $\alpha^{n} \geq 3$ for $n \geq 1$, we can make the following observations:

- $\mathcal{T}_{\text {both corrections }}^{\text {s-channel }}$ blows up as $s \rightarrow-\infty$.

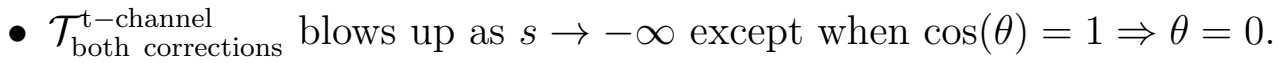

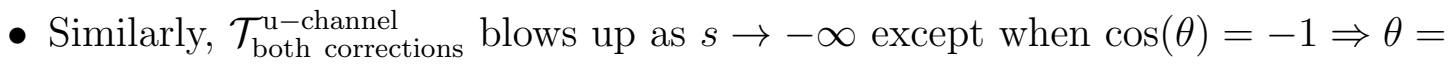
$\pi$.

Thus, one can check that the cross section $\sigma_{\text {both }}$ corrections corresponding to $\mathcal{T}_{\text {both }}$ corrections $=$ $\mathcal{T}_{\text {both corrections }}^{\text {s-chanel }}+\mathcal{T}_{\text {both corrections }}^{\mathrm{t}}+\mathcal{T}_{\text {both corrections }}^{\mathrm{u} \text {-channel }}$ blows up as $s \rightarrow-\infty$.

We see that dressing the vertices by making propagator and vertex loop corrections to the bare vertices cannot ameliorate the UV external momentum growth of scattering diagrams in our toy model example, Eq. (2.1). This motivates us to consider something very different; in the following section, we shall not consider a finite-order, higher-derivative theory, but an infinite-derivative massless scalar field theory with cubic interaction in $\phi$. Both the free and interaction parts of the action will contain infinite derivatives.

\section{$3 \quad$ Scatterings in infinite-derivative theory}

We saw in section 2 that, within the context of a finite-order higher-derivative scalar toy model, we cannot tame the UV external momentum growth appearing in scattering diagrams. In particular, we need to "soften" the external momentum contributions coming from the dressed vertices; as we saw in subsections $2.2 \& 2.3$, dressing the vertices in a finite-order higher-derivative toy model cannot help us tame the external momentum growth of the scattering diagrams. Since this is not possible for a finiteorder higher-derivative toy model, we shall examine an infinite-derivative scalar toy model. Therefore, let us consider the following action, which has a cubic interaction where $\lambda \ll \mathcal{O}(1)$, and treat it perturbatively:

$$
S=S_{\text {free }}+S_{\text {int }}
$$

where

$$
S_{\text {free }}=\frac{1}{2} \int d^{4} x(\phi \square a(\square) \phi)
$$


and

$$
S_{\text {int }}=\lambda \int d^{4} x(\phi \square \phi a(\square) \phi) .
$$

Now, let us demand that the propagator for free action retains only the massless scalar degree of freedom. In which case, we assume that the kinetic term obtains an entire function correction. For simplicity, we take such a function to be Gaussian:

$$
a(\square)=e^{-\square / M^{2}},
$$

where $M$ is the mass scale at which the non-local modifications become important. With this choice, the infinite-derivative theory can be made ghost-free [38, 39]. Such a choice of $a(\square)$ is also well motivated by $p$-adic strings [43]. The propagator in momentum space is then given in the Euclidean space, by

$$
\Pi\left(k^{2}\right)=\frac{-i}{k^{2} e^{\bar{k}^{2}}},
$$

where barred 4 -momentum vectors denote $\bar{k}=k / M$. The vertex factor for three incoming momenta $k_{1}, k_{2}, k_{3}$ satisfying the following conservation law,

$$
k_{1}+k_{2}+k_{3}=0
$$

is given by

$$
\lambda V\left(k_{1}, k_{2}, k_{3}\right)=-i \lambda\left[k_{1}^{2}\left(e^{\bar{k}_{2}^{2}}+e^{\bar{k}_{3}^{2}}\right)+k_{2}^{2}\left(e^{\bar{k}_{3}^{2}}+e^{\bar{k}_{1}^{2}}\right)+k_{3}^{2}\left(e^{\bar{k}_{1}^{2}}+e^{\bar{k}_{2}^{2}}\right)\right] .
$$

We can compute the tree-level $s, t, u$ channels in the CM frame and we obtain

$$
\begin{gathered}
i \mathcal{T}_{\text {tree-level }}^{\text {s-channel }}=-\lambda^{2} s^{2}\left[3 e^{-s / 2 M^{2}}+e^{-s / M^{2}}\right]^{2}\left(\frac{i}{s e^{-s / M^{2}}}\right), \\
i \mathcal{T}_{\text {tree-level }}^{\mathrm{t}-\text { channel }}=-\lambda^{2}\left[(s+2 t) e^{-s / 2 M^{2}}+s e^{-t / M^{2}}\right]^{2}\left(\frac{i}{t e^{-t / M^{2}}}\right), \\
i \mathcal{T}_{\text {tree-level }}^{\mathrm{u}-\text { channel }}=-\lambda^{2}\left[(s+2 u) e^{-s / 2 M^{2}}+s e^{-u / M^{2}}\right]^{2}\left(\frac{i}{u e^{-u / M^{2}}}\right) .
\end{gathered}
$$

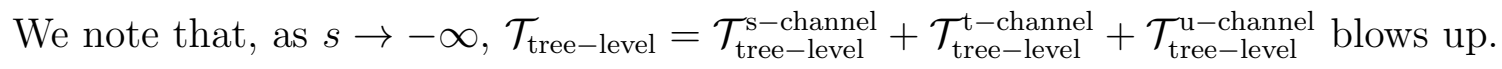

Now, in order to compute the dressed propagator, we have, first, to write down the 1-loop, 2-point function with external momenta $p$ and $-p$. We have

$$
\begin{aligned}
\Gamma_{2,1}\left(p^{2}\right) & =\frac{i \lambda^{2}}{2} \int \frac{d^{4} k}{(2 \pi)^{4}} \frac{1}{\left(\frac{p}{2}+k\right)^{2}\left(\frac{p}{2}-k\right)^{2} e^{\left(\frac{\bar{p}}{2}+\bar{k}\right)^{2}} e^{\left(\frac{\bar{p}}{2}-\bar{k}\right)^{2}}} \\
& \times\left[p^{2}\left(e^{\left(\frac{\bar{p}}{2}+\bar{k}\right)^{2}}+e^{\left(\frac{\bar{p}}{2}-\bar{k}\right)^{2}}\right)+\left(\frac{p}{2}+k\right)^{2}\left(e^{\bar{p}^{2}}+e^{\left(\frac{\bar{p}}{2}-\bar{k}\right)^{2}}\right)+\left(\frac{p}{2}-k\right)^{2}\left(e^{\bar{p}^{2}}+e^{\left(\frac{\bar{p}}{2}+\bar{k}\right)^{2}}\right)\right]^{2} .
\end{aligned}
$$


After renormalising the divergent (in terms of the internal loop momentum $k^{\mu}$ ) terms 8 , we have that the most divergent part (in terms of the external momentum $p^{\mu}$ ) of the 1-loop, 2-point function is given by

$$
\frac{i \lambda^{2} M^{4} e^{\frac{3 \bar{p}^{2}}{2}}\left(4 M^{2}+p^{2}\right)}{32 \pi^{2} p^{2}} .
$$

Thus, the renormalised 1-loop, 2-point function goes as $\left(1+4 \bar{p}^{-2}\right) e^{\frac{3 \bar{p}^{2}}{2}}$ when $p$ is large. As $s \rightarrow-\infty, \Gamma_{2,1 \mathrm{r}}(-s)$ goes as $e^{-\frac{3 s}{2 M^{2}}}$. Since

$$
\begin{aligned}
i \mathcal{T}_{1-\text { loop }} & =\lambda^{2}\left(p_{1}, p_{2},-p_{1}-p_{2}\right) V\left(-p_{3},-p_{4}, p_{1}+p_{2}\right)\left(\frac{i}{s e^{-s / M^{2}}}\right)^{2} \Gamma_{2,1 \mathrm{r}}(-s) \\
& +\lambda^{2} V\left(p_{1},-p_{3}, p_{3}-p_{1}\right) V\left(p_{2},-p_{4}, p_{1}-p_{3}\right)\left(\frac{i}{t e^{-t / M^{2}}}\right)^{2} \Gamma_{2,1 \mathrm{r}}(-t) \\
& +\lambda^{2} V\left(p_{1},-p_{4}, p_{4}-p_{1}\right) V\left(p_{2},-p_{3}, p_{1}-p_{4}\right)\left(\frac{i}{u e^{-u / M^{2}}}\right)^{2} \Gamma_{2,1 \mathrm{r}}(-u),
\end{aligned}
$$

we have that the $s$-channel of $\mathcal{T}_{1-\text { loop }}$, see again Fig. 4 , goes as $e^{-\frac{2 s}{M^{2}}} e^{\frac{2 s}{M^{2}}} e^{-\frac{3 s}{2 M^{2}}}=e^{-\frac{3 s}{2 M^{2}}}$

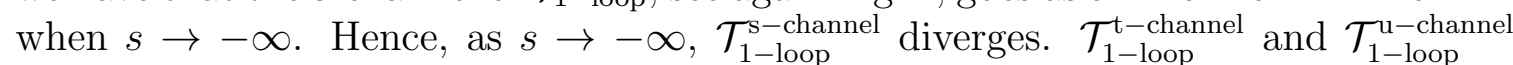
also diverge as $s \rightarrow-\infty$.

\subsection{Dressing the propagator}

Since for large $p$, the dressed propagator goes as

$$
\widetilde{\Pi}\left(p^{2}\right) \approx\left(1+4 \bar{p}^{-2}\right)^{-1} e^{-\frac{3 \bar{p}^{2}}{2}}
$$

we observe that the dressed propagator is more strongly exponentially suppressed than the bare propagator.

Since

$$
\begin{aligned}
i \mathcal{T}_{\text {dressed }} & =\lambda^{2} V\left(p_{1}, p_{2},-p_{1}-p_{2}\right) V\left(-p_{3},-p_{4}, p_{1}+p_{2}\right) \widetilde{\Pi}(-s) \\
& +\lambda^{2} V\left(p_{1},-p_{3}, p_{3}-p_{1}\right) V\left(p_{2},-p_{4}, p_{1}-p_{3}\right) \widetilde{\Pi}(-t) \\
& +\lambda^{2} V\left(p_{1},-p_{4}, p_{4}-p_{1}\right) V\left(p_{2},-p_{3}, p_{1}-p_{4}\right) \widetilde{\Pi}(-u)
\end{aligned}
$$

then, if we now replace the bare propagator with the dressed propagator in the tree-

\footnotetext{
${ }^{8}$ Within the context of dimensional regularisation, we obtain an $\epsilon^{-1}$ pole, where $\epsilon=4-d$ and $d$ is the number of dimensions.
} 
level scattering diagrams, see Fig. 5 (bottom), we will have, as $s \rightarrow-\infty$,

$$
\begin{aligned}
& \mathcal{T}_{\text {dressed }}^{\text {s-channel }} \sim\left[3 e^{-\frac{s}{2 M^{2}}}+e^{-\frac{s}{M^{2}}}\right]^{2} e^{\frac{3 s}{2 M^{2}}} \sim e^{-\frac{s}{2 M^{2}}}, \\
& \mathcal{T}_{\text {dressed }}^{\mathrm{t}-\text { channel }} \sim\left[(s+2 t) e^{-\frac{s}{2 M^{2}}}+s e^{-\frac{t}{M^{2}}}\right]^{2} e^{\frac{3 t}{2 M^{2}}}=\left[s(2-\cos \theta) e^{-\frac{s}{2 M^{2}}}+s e^{-\frac{s(1-\cos \theta)}{2 M^{2}}}\right]^{2} e^{\frac{3 s(1-\cos \theta)}{4 M^{2}}}, \\
& \mathcal{T}_{\text {dressed }}^{\mathrm{u}-\text { channel }} \sim\left[(s+2 u) e^{-\frac{s}{2 M^{2}}}+s e^{-\frac{u}{M^{2}}}\right]^{2} e^{\frac{3 u}{2 M^{2}}}=\left[s(2+\cos \theta) e^{-\frac{s}{2 M^{2}}}+s e^{-\frac{s(1+\cos \theta)}{2 M^{2}}}\right]^{2} e^{\frac{3 s(1+\cos \theta)}{4 M^{2}}} .
\end{aligned}
$$

Hence, we can make the following observations:

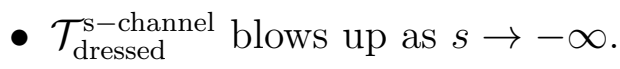

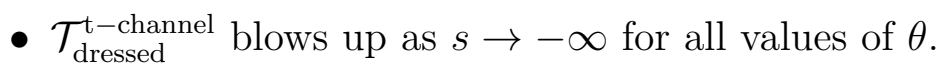

- $\mathcal{T}_{\text {dressed }}^{\mathrm{u}-\text { channel }}$ blows up as $s \rightarrow-\infty$ for all values of $\theta$.

Since $\mathcal{T}_{\text {dressed }}=\mathcal{T}_{\text {dressed }}^{\mathrm{s}-\text { channel }}+\mathcal{T}_{\text {dressed }}^{\mathrm{t}-\text { channel }}+\mathcal{T}_{\text {dressed }}^{\mathrm{u} \text {-chanel }}$, one can verify that the total cross section $\sigma_{\text {dressed }}$ corresponding to $\mathcal{T}_{\text {dressed }}$ blows up as $s \rightarrow-\infty$. We also observe that the external momentum dependence of $\mathcal{T}_{\text {dressed }}$ exhibits less growth for large external momenta as compared to the external momentum dependence of $\mathcal{T}_{\text {tree-level }}$ (or $\mathcal{T}_{1-\text { loop }}$ ).

To conclude, the use of the dressed propagator ameliorates the external momentum growth of the scattering diagrams, but this is not sufficient by itself. In subsection 3.2, we shall dress the vertices to see whether we can eliminate the external momentum growth of the scattering diagrams.

\subsection{Dressing the vertices by making vertex loop corrections to the bare vertices}

In this subsection, we shall dress the vertices by making renormalised vertex loop corrections to the bare vertices at the left- and right-ends of the scattering diagrams, see Fig. 7. We have that both the bare propagators and the bare vertices can be written as exponentials in momenta; after integration with respect to the internal loop momentum $k$, we obtain an exponential expression where the exponents are in terms of the external momenta $p_{1}, p_{2}, p_{3}$. As the loop-order increases, the 3-point function can still be written as an exponential function of the external momenta; this happens because, as previously, the (bare) propagators are exponentials in momenta while the (dressed) vertices are also exponentials in momenta.Thus, in the UV limit, i.e., as $p_{i} \rightarrow \infty$, where $i=1,2,3$, the 3 -point function, again see Fig. 7, can be written as

$$
\Gamma_{3} \stackrel{U V}{\longrightarrow} \sum_{\alpha, \beta, \gamma} e^{\alpha \bar{p}_{1}^{2}+\beta \bar{p}_{2}^{2}+\gamma \bar{p}_{3}^{2}}
$$


with the convention

$$
\alpha \geq \beta \geq \gamma
$$

where $p_{1}, p_{2}, p_{3}$ are the three external momenta. The reason we expect the external momentum dependence of the 3-point function to be given by Eq. (3.18) is that, once all the (lower-) loop subdiagrams have been integrated out, what remains are exponential expressions in terms of the three external momenta $p_{1}, p_{2}, p_{3}$.

The best way to obtain the largest exponents for the external momenta is to have the $\alpha$ exponent correspond to the external momenta. Assuming a symmetric distribution of $(\beta, \gamma)$ among the internal loops and considering the $n$-loop, 3-point diagram with symmetrical routing of momenta, see Fig. 7, the propagators in the 1-loop triangle are given by

$$
e^{-\left(\bar{k}+\frac{\bar{p}_{1}}{3}-\frac{\bar{p}_{2}}{3}\right)^{2}}, e^{-\left(\bar{k}+\frac{\bar{p}_{2}}{3}-\frac{\bar{p}_{3}}{3}\right)^{2}}, e^{-\left(\bar{k}+\frac{\bar{p}_{3}}{3}-\frac{\bar{p}_{1}}{3}\right)^{2}},
$$

and the vertex factors are

$$
\begin{aligned}
& e^{\alpha^{n-1} \bar{p}_{1}^{2}+\beta^{n-1}\left(\bar{k}+\frac{\bar{p}_{3}}{3}-\frac{\bar{p}_{1}}{3}\right)^{2}+\gamma^{n-1}\left(\bar{k}+\frac{\bar{p}_{1}}{3}-\frac{\bar{p}_{2}}{3}\right)^{2},} \\
& e^{\alpha^{n-1} \bar{p}_{2}^{2}+\beta^{n-1}\left(\bar{k}+\frac{\bar{p}_{1}}{3}-\frac{\bar{p}_{2}}{3}\right)^{2}+\gamma^{n-1}\left(\bar{k}+\frac{\bar{p}_{2}}{3}-\frac{\bar{p}_{3}}{3}\right)^{2}}, \\
& e^{\alpha^{n-1} \bar{p}_{3}^{2}+\beta^{n-1}\left(\bar{k}+\frac{\bar{p}_{2}}{3}-\frac{\bar{p}_{3}}{3}\right)^{2}+\gamma^{n-1}\left(\bar{k}+\frac{\bar{p}_{3}}{3}-\frac{\bar{p}_{1}}{3}\right)^{2}} \text {. }
\end{aligned}
$$

Conservation of momenta then yields, in the UV, i.e., as $p_{i} \rightarrow \infty$, where $i=1,2,3$,

$$
\begin{aligned}
\Gamma_{3, n} & \longrightarrow \int \frac{d^{4} k}{(2 \pi)^{4}}\left[\frac{e^{\alpha^{n-1} \bar{p}_{1}^{2}+\beta^{n-1}\left(\bar{k}+\frac{\bar{p}_{3}}{3}-\frac{\bar{p}_{1}}{3}\right)^{2}+\gamma^{n-1}\left(\bar{k}+\frac{\bar{p}_{1}}{3}-\frac{\bar{p}_{2}}{3}\right)^{2}}}{e^{-\left(\bar{k}+\frac{\bar{p}_{1}}{3}-\frac{\bar{p}_{2}}{3}\right)^{2}} e^{-\left(\bar{k}+\frac{\bar{p}_{2}}{3}-\frac{\bar{p}_{3}}{3}\right)^{2}} e^{-\left(\bar{k}+\frac{\bar{p}_{3}}{3}-\frac{\bar{p}_{1}}{3}\right)^{2}}}\right] \\
& \left.\times e^{\alpha^{n-1} \bar{p}_{2}^{2}+\beta^{n-1}\left(\bar{k}+\frac{\bar{p}_{1}}{3}-\frac{\bar{p}_{2}}{3}\right)^{2}+\gamma^{n-1}\left(\bar{k}+\frac{\bar{p}_{2}}{3}-\frac{\bar{p}_{3}}{3}\right)^{2}} e^{\alpha^{n-1} \bar{p}_{3}^{2}+\beta^{n-1}\left(\bar{k}+\frac{\bar{p}_{2}}{3}-\frac{\bar{p}_{3}}{3}\right)^{2}+\gamma^{n-1}\left(\bar{k}+\frac{\bar{p}_{3}}{3}-\frac{\bar{p}_{1}}{3}\right)^{2}}\right] \\
& =\int \frac{d^{4} k}{(2 \pi)^{4}} \frac{e^{\alpha^{n-1}\left(\bar{p}_{1}^{2}+\bar{p}_{2}^{2}+\bar{p}_{3}^{2}\right)}}{e^{\left[1-\beta^{n-1}-\gamma^{n-1}\right]\left[3 \bar{k}^{2}+\frac{1}{3}\left(\bar{p}_{1}^{2}+\bar{p}_{2}^{2}+\bar{p}_{3}^{2}\right)\right]}},
\end{aligned}
$$

where $p_{1}, p_{2}, p_{3}$ are the external momenta for the 1-loop triangle, and the superscript in the $\alpha, \beta, \gamma$ indicates that these are coefficients that one obtains from contributions up to $n-1$ loop level.

Integrating Eq. (3.22) with respect to the loop momentum $k$ and reminding ourselves that $\alpha^{n}, \beta^{n}$ and $\gamma^{n}$ are the coefficients of $\bar{p}_{1}^{2}, \bar{p}_{2}^{2}$ and $\bar{p}_{3}^{2}$, respectively, appearing in the exponentials in Eq. (3.18), we have

$$
\alpha^{n}=\beta^{n}=\gamma^{n}=\alpha^{n-1}+\frac{1}{3}\left(\beta^{n-1}+\gamma^{n-1}\right)-\frac{1}{3} .
$$

In particular, for the 1-loop, 3-point graph, one has to use the 3-point bare vertices (see Eq. (3.7)): $\alpha^{0}=1$ and $\beta^{0}=\gamma^{0}=0$. One then obtains

$$
\alpha^{1}=\beta^{1}=\gamma^{1}=\frac{2}{3},
$$


leading to an overall symmetric vertex: $e^{\frac{2}{3}\left(\bar{p}_{1}^{2}+\bar{p}_{2}^{2}+\bar{p}_{3}^{2}\right)}$ and $\alpha^{1}+\beta^{1}+\gamma^{1}=2$. We observe that, as $n$ increases, $\alpha^{n}, \beta^{n}$ and $\gamma^{n}$ increase; this means that, as the number of loops increases, the external momentum contributions of the dressed vertices become larger and larger.

We conclude that dressing the vertices by considering just vertex loop corrections to the bare vertices does not ameliorate the external momentum growth of scattering diagrams in the UV in our toy model example Eq. (3.1); in fact, it makes that growth increase. In the next subsection, we shall dress the vertices by considering both propagator and vertex loop corrections to the bare vertices.

\subsection{Dressing the vertices by making propagator \& vertex loop corrections to the bare vertices}

As our next step, let us now consider $\mathcal{T}_{\text {dressed }} 9$, We know that $\mathcal{T}_{\text {dressed }}$ diverges as $s \rightarrow-\infty$. Let us now dress the vertices by making renormalised propagator and vertex loop corrections to the bare vertices at the left- and right-ends of the diagram. Regarding the dressed propagator, we have $\widetilde{\Pi}\left(p^{2}\right) \stackrel{U V}{\longrightarrow} e^{-3 \bar{p}^{2} / 2}$. Therefore, following the prescription given in section 3.2 , the 3 -point function can again be written as an exponential function of the external momenta; this happens because, as previously, the (dressed) propagators are exponentials in momenta while the (dressed) vertices are also exponentials in momenta. Hence, in the UV limit, i.e., as $p_{i} \rightarrow \infty$, where $i=1,2,3$, the 3-point function $\Gamma_{3}$, see Fig. 8, is again given by Eq. (3.18). As previously, the best way to obtain the largest exponents for the external momenta is to have the $\alpha$ exponent correspond to the external momenta. Assuming a symmetric distribution of $(\beta, \gamma)$ among the internal loops and considering the $n$-loop, 3-point diagram with symmetrical routing of momenta, see Fig. 8 , the propagators in the 1-loop triangle are given by

$$
e^{-\frac{3}{2}\left(\bar{k}+\frac{\bar{p}_{1}}{3}-\frac{\bar{p}_{2}}{3}\right)^{2}}, e^{-\frac{3}{2}\left(\bar{k}+\frac{\bar{p}_{2}}{3}-\frac{\bar{p}_{3}}{3}\right)^{2}}, e^{-\frac{3}{2}\left(\bar{k}+\frac{\bar{p}_{3}}{3}-\frac{\bar{p}_{1}}{3}\right)^{2}},
$$

and the vertex factors are

$$
\begin{aligned}
& e^{\alpha^{n-1} \bar{p}_{1}^{2}+\beta^{n-1}\left(\bar{k}+\frac{\bar{p}_{3}}{3}-\frac{\bar{p}_{1}}{3}\right)^{2}+\gamma^{n-1}\left(\bar{k}+\frac{\bar{p}_{1}}{3}-\frac{\bar{p}_{2}}{3}\right)^{2},} \\
& e^{\alpha^{n-1} \bar{p}_{2}^{2}+\beta^{n-1}\left(\bar{k}+\frac{\bar{p}_{1}}{3}-\frac{\bar{p}_{2}}{3}\right)^{2}+\gamma^{n-1}\left(\bar{k}+\frac{\bar{p}_{2}}{3}-\frac{\bar{p}_{3}}{3}\right)^{2}}, \\
& e^{\alpha^{n-1} \bar{p}_{3}^{2}+\beta^{n-1}\left(\bar{k}+\frac{\bar{p}_{2}}{3}-\frac{\bar{p}_{3}}{3}\right)^{2}+\gamma^{n-1}\left(\bar{k}+\frac{\bar{p}_{3}}{3}-\frac{\bar{p}_{1}}{3}\right)^{2}} \text {. }
\end{aligned}
$$

\footnotetext{
${ }^{9}$ We could equally well consider $\mathcal{T}_{\text {tree-level }}, \mathcal{T}_{1 \text {-loop }}$, etc. By making renormalised propagator \& vertex loop corrections to the bare vertices at the left- and right-ends of the scattering diagram under consideration, the external momentum growth would be eliminated at sufficiently high loop order.
} 
In the UV, i.e., as $p_{i} \rightarrow \infty$, where $i=1,2,3$, conservation of momenta gives

$$
\begin{aligned}
\Gamma_{3, n} & \longrightarrow \int \frac{d^{4} k}{(2 \pi)^{4}} \frac{e^{\alpha^{n-1} \bar{p}_{1}^{2}+\beta^{n-1}\left(\bar{k}+\frac{\bar{p}_{3}}{3}-\frac{\bar{p}_{1}}{3}\right)^{2}+\gamma^{n-1}\left(\bar{k}+\frac{\bar{p}_{1}}{3}-\frac{\bar{p}_{2}}{3}\right)^{2}}}{e^{-\frac{3}{2}\left(\bar{k}+\frac{\bar{p}_{1}}{3}-\frac{\bar{p}_{2}}{3}\right)^{2}} e^{-\frac{3}{2}\left(\bar{k}+\frac{\bar{p}_{2}}{3}-\frac{\bar{p}_{3}}{3}\right)^{2}} e^{-\frac{3}{2}\left(\bar{k}+\frac{\bar{p}_{3}}{3}-\frac{\bar{p}_{1}}{3}\right)^{2}}} \\
& \times e^{\alpha^{n-1} \bar{p}_{2}^{2}+\beta^{n-1}\left(\bar{k}+\frac{\bar{p}_{1}}{3}-\frac{\bar{p}_{2}}{3}\right)^{2}+\gamma^{n-1}\left(\bar{k}+\frac{\bar{p}_{2}}{3}-\frac{\bar{p}_{3}}{3}\right)^{2}} e^{\alpha^{n-1} \bar{p}_{3}^{2}+\beta^{n-1}\left(\bar{k}+\frac{\bar{p}_{2}}{3}-\frac{\bar{p}_{3}}{3}\right)^{2}+\gamma^{n-1}\left(\bar{k}+\frac{\bar{p}_{3}}{3}-\frac{\bar{p}_{1}}{3}\right)^{2}} \\
& =\int \frac{d^{4} k}{(2 \pi)^{4}} \frac{e^{\alpha^{n-1}\left(\bar{p}_{1}^{2}+\bar{p}_{2}^{2}+\bar{p}_{3}^{2}\right)}}{e^{\left[\frac{3}{2}-\beta^{n-1}-\gamma^{n-1}\right]\left[3 \bar{k}^{2}+\frac{1}{3}\left(\bar{p}_{1}^{2}+\bar{p}_{2}^{2}+\bar{p}_{3}^{2}\right)\right]}}
\end{aligned}
$$

where $p_{1}, p_{2}, p_{3}$ are the external momenta for the 1-loop triangle, and the superscript in the $\alpha, \beta, \gamma$ indicates that these are coefficients that one obtains from contributions up to $n-1$ loop level.

After integrating Eq. (3.27) with respect to the loop momentum $k$, one obtains

$$
\alpha^{n}=\beta^{n}=\gamma^{n}=\alpha^{n-1}+\frac{1}{3}\left(\beta^{n-1}+\gamma^{n-1}\right)-\frac{1}{2} .
$$

For the 3-point bare vertices, we have $\alpha^{0}=1$ and $\beta^{0}=\gamma^{0}=0$. Employing Eq. 3.28), one then obtains

$$
\alpha^{1}=\beta^{1}=\gamma^{1}=\frac{1}{2} .
$$

We observe that $\alpha^{1}+\beta^{1}+\gamma^{1}=\frac{3}{2}$. We anticipate that the exponents become smaller as the loop order becomes larger; hence, we posit that the following inequality holds:

$$
\alpha^{n}+\beta^{n}+\gamma^{n} \leq \frac{3}{2} .
$$

Using Eq. (3.28), we see that Eq. (3.30) is satisfied as long as the following condition is also satisfied:

$$
\alpha^{n-1}+\frac{1}{3}\left(\beta^{n-1}+\gamma^{n-1}\right) \leq 1
$$

To recap, we have shown that if, up to loop order $n-1$, Eq. (3.31) holds, then, at loop order $n$, Eq. (3.30) holds too. In order to conclude the recursive argument (see [59] for more details regarding the recursive argument), we have to show that Eq. (3.31) holds at loop order $n$ as well. Consequently, we have

$$
\alpha^{n}+\frac{1}{3}\left(\beta^{n}+\gamma^{n}\right)=\frac{5}{3}\left[\alpha^{n-1}+\frac{1}{3}\left(\beta^{n-1}+\gamma^{n-1}\right)-\frac{1}{2}\right] \leq \frac{5}{6}<1 .
$$

We have verified that Eq. (3.31) does hold at loop order $n$. As a result, the loops stay finite as the loop order increases.

Now, since $\alpha^{1}=\beta^{1}=\gamma^{1}=\frac{1}{2}$, and using Eq. (3.28), we obtain that, for $n=2$,

$$
\alpha^{2}=\beta^{2}=\gamma^{2}=\frac{1}{3},
$$

for $n=3$,

$$
\alpha^{3}=\beta^{3}=\gamma^{3}=\frac{1}{18}
$$


for $n=4$,

$$
\alpha^{4}=\beta^{4}=\gamma^{4}=-\frac{11}{27}
$$

We conclude that, for $n \geq 4, \alpha^{n}, \beta^{n}$ and $\gamma^{n}$ become negative. The fact that $\alpha^{n}, \beta^{n}$ and $\gamma^{n}$ become negative for sufficiently large $n$ should be emphasised since it is precisely this negativity which eliminates the external momentum growth of the scattering diagrams in the UV.

For $n=4$, we have the following results:

- We find that the largest external momentum contribution of the $s$-channel, see Fig. 9, goes as

$$
e^{\frac{44 s}{27 M^{2}}} e^{\frac{3 s}{2 M^{2}}}=e^{\frac{169 s}{54 M^{2}}},
$$

which tends to 0 as $s \rightarrow-\infty$.

- Regarding the $t$-channel, the largest external momentum contribution goes as

$$
e^{\frac{22 t}{27 M^{2}}} e^{\frac{22 s}{27 M^{2}}} e^{\frac{3 t}{2 M^{2}}}=e^{\frac{s(213-125 \cos \theta)}{108 M^{2}}},
$$

which, again, tends to 0 as $s \rightarrow-\infty$ for all values of $\theta$.

- Regarding the $u$-channel, the largest external momentum contribution goes as

$$
e^{\frac{22 u}{27 M^{2}}} e^{\frac{22 s}{27 M^{2}}} e^{\frac{3 u}{2 M^{2}}}=e^{\frac{s(213+125 \cos \theta)}{108 M^{2}}},
$$

which, again, tends to 0 as $s \rightarrow-\infty$ for all values of $\theta$. Hence, for sufficiently large $n$ (specifically, for $n \geq 4$ ), there is no exponential growth for the $s$-, $t$ - and $u$-channels as $s \rightarrow-\infty$.

Let us also point out that we do not have to worry about polynomial growth in $s$ since any polynomial functions of $s$ will be multiplied by exponential functions of $s$ and their product will tend to 0 as $s \rightarrow-\infty$, keeping in mind that exponential functions always dominate polynomial ones at large values.

Dressing the vertices by making both propagator and vertex loop corrections to the bare vertices ameliorates and, in fact, completely eliminates, for sufficiently large $n$, the external momentum growth of the scattering diagrams in the UV. In the next section, we will study an infinite-derivative scalar toy model inspired by a ghost-free and singularity-free theory of gravity.

\section{Scattering in infinite-derivative theories of grav- ity}

Inspired by the results of previous section, let us now investigate scattering diagrams in the context of infinite-derivative theories of gravity, which is ghost-free and 
singularity-free, for brevity we call it BGKM gravity [38]. In [59], we studied the quantum loops for an infinite-derivative scalar field theory action as a toy model to mimic the UV properties of the BGKM gravity. Expanding the BGKM action around the Minkowski vacuum 10 , one can obtain, for instance, the "free" part that determines the propagator from the $\mathcal{O}\left(h^{2}\right)$ terms; $h_{\mu \nu}$ denotes a small perturbation around Minkowski spacetime: $g_{\mu \nu}=\eta_{\mu \nu}+h_{\mu \nu}$. The $\mathcal{O}\left(h^{3}\right)$ terms determine cubic interaction vertices. Unfortunately, $\mathcal{O}\left(h^{3}\right)$ terms are technically challenging and some of the expressions involve double sums. Instead of getting involved with too many technicalities, we shall, therefore, choose to work with a simple toy model action that respects a combination of shift and scaling symmetry at the level of equation of motion. This will allow us to capture some of the essential features of BGKM gravity, such as the compensating nature of exponential suppression in the propagator and an exponential enhancement in the vertex factor.

The infinite-derivative action that can modify the propagator of the graviton without introducing any new states is of the form [38]

$$
S=S_{E H}+S_{Q}
$$

where $S_{E H}$ is the Einstein-Hilbert action,

$$
\int d^{4} x \sqrt{-g} \frac{R}{2}
$$

and $S_{Q}$ is given by

$$
S_{Q}=\int d^{4} x \sqrt{-g}\left[R \mathcal{F}_{1}(\square) R+R_{\mu \nu} \mathcal{F}_{2}(\square) R^{\mu \nu}+R_{\mu \nu \lambda \sigma} \mathcal{F}_{3}(\square) R^{\mu \nu \lambda \sigma}\right],
$$

where the $\mathcal{F}_{i}$ 's are analytic functions of $\square$ (the covariant d'Alembertian operator):

$$
\mathcal{F}_{i}(\square)=\sum_{n=0}^{\infty} f_{i_{n}} \square^{n},
$$

satisfying

$$
2 \mathcal{F}_{1}+\mathcal{F}_{2}+2 \mathcal{F}_{3}=0
$$

and the constraint that the combination

$$
a(\square)=1-\frac{1}{2} \mathcal{F}_{2}(\square) \square-2 \mathcal{F}_{3}(\square) \square
$$

is an entire function with no zeroes. In Eq. (4.4), the $f_{i_{n}}$ 's are real coefficients. Eqs. (4.1)-(4.6) define the BGKM gravity models. For BGKM gravity, we have the propagator [38, 39],

$$
\Pi\left(k^{2}\right)=-\frac{i}{k^{2} a\left(-k^{2}\right)}\left(\mathcal{P}^{2}-\frac{1}{2} \mathcal{P}_{s}^{0}\right)=\frac{1}{a\left(-k^{2}\right)} \Pi_{G R},
$$

\footnotetext{
${ }^{10}$ One could expand the BGKM action and, subsequently, derive the propagator for a different background metric such as (A)dS [45]. Computing graviton-graviton scattering diagrams in (A)dS spacetime is a topic for future investigation.
} 
for the physical degrees of freedom for a graviton propagating in 4 dimensions; see [38, 39, 60] for the definitions of the spin projector operators $\mathcal{P}^{2}$ and $\mathcal{P}_{s}^{0}$.

Since we know that the field equations of GR exhibit a global scaling symmetry,

$$
g_{\mu \nu} \rightarrow \lambda g_{\mu \nu}
$$

When we expand the metric around the Minkowski vacuum,

$$
g_{\mu \nu}=\eta_{\mu \nu}+h_{\mu \nu}
$$

the scaling symmetry translates to a symmetry for $h_{\mu \nu}$, whose infinitesimal version is given by

$$
h_{\mu \nu} \rightarrow(1+\epsilon) h_{\mu \nu}+\epsilon \eta_{\mu \nu} .
$$

The symmetry relates the free and interaction terms just like gauge symmetry does. Thus, we are going to use this combination of shift and scaling symmetry,

$$
\phi \rightarrow(1+\epsilon) \phi+\epsilon
$$

to arrive at a scalar toy model, whose propagator and vertices preserve the compensating nature found in the full BGKM gravity. Now, let us write down explicitly the scalar toy model action and the Feynman rules for that action, i.e., the propagator and the vertex factors. Our scalar toy model action is given by:

$$
S_{\text {scalar }}=S_{\text {free }}+S_{\text {int }}
$$

where

$$
S_{\text {free }}=\frac{1}{2} \int d^{4} x(\phi \square a(\square) \phi)
$$

and

$$
S_{\text {int }}=\frac{1}{M_{P}} \int d^{4} x\left(\frac{1}{4} \phi \partial_{\mu} \phi \partial^{\mu} \phi+\frac{1}{4} \phi \square \phi a(\square) \phi-\frac{1}{4} \phi \partial_{\mu} \phi a(\square) \partial^{\mu} \phi\right) .
$$

For the purpose of this paper, we are going to choose:

$$
a(\square)=e^{-\square / M^{2}},
$$

where $M$ is the mass scale at which the non-local modifications become important. The propagator in momentum space for Eq. (4.13) is then given by

$$
\Pi\left(k^{2}\right)=\frac{-i}{k^{2} e^{\bar{k}^{2}}},
$$

where barred 4-momentum vectors from now on will denote the momentum divided by the mass scale $M$. The vertex factor for three incoming momenta $k_{1}, k_{2}, k_{3}$ satisfying the conservation law:

$$
k_{1}+k_{2}+k_{3}=0
$$


is then given by

$$
\frac{1}{M_{P}} V\left(k_{1}, k_{2}, k_{3}\right)=\frac{i}{M_{P}} C\left(k_{1}, k_{2}, k_{3}\right)\left[1-e^{\bar{k}_{1}^{2}}-e^{\bar{k}_{2}^{2}}-e^{\bar{k}_{3}^{2}}\right],
$$

where

$$
C\left(k_{1}, k_{2}, k_{3}\right)=\frac{1}{4}\left(k_{1}^{2}+k_{2}^{2}+k_{3}^{2}\right) .
$$

For the above set-up, 1-loop, 2-point diagram, both with zero and arbitrary external momenta have been computed in Ref. [59], which gives a $\Lambda^{4}$ divergence, where $\Lambda$ is a momentum cut-off. Further, 1-loop, $N$-point diagrams with vanishing external momenta were also computed. The 2-loop diagrams with zero external momenta also give a $\Lambda^{4}$ divergence, suggesting that we do not get new divergences as we proceed from 1-loop to 2-loop. In Ref. [59], the authors have computed 1-loop and 2-loop computations with external momenta and paid extra care in understanding the 1loop, 2-point function which appeared as a subdivergence in higher-loop diagrams.

Typically, in the 1-loop, 2-point function, the authors obtained $e^{\frac{3 \bar{p}^{2}}{2}}$ external momentum dependence in the UV, which indicates that, for $\bar{p}^{2} \rightarrow \infty$, the 1-loop, 2-point function tends to infinity. This may appear as an initial setback, but, actually, this external momentum dependence is what, we believe, makes all higher-loop and higherpoint diagrams finite once the bare propagators were replaced by dressed propagators. The dressed propagator is given by (see Ref. [59])

$$
\widetilde{\Pi}\left(p^{2}\right)=\frac{\Pi\left(p^{2}\right)}{1-\Pi\left(p^{2}\right) \Gamma_{2,1 \mathrm{r}}\left(p^{2}\right)}=\frac{-i}{p^{2} e^{\bar{p}^{2}}-\frac{M^{4}}{M_{P}^{2}} f\left(\bar{p}^{2}\right)},
$$

where $f\left(\bar{p}^{2}\right)$ grows as $e^{\frac{3 \bar{p}^{2}}{2}}$ as $\bar{p}^{2} \rightarrow \infty$. For such an external momentum dependence, the dressed propagator is more strongly suppressed than the bare one. The finiteness of all higher-loop and higher-point diagrams became possible because the exponential suppression in the dressed propagator, which is $e^{-\frac{3 \bar{p}^{2}}{2}}$ in the UV, overcame the exponential enhancement arising from the vertices. The 1-loop, $N$-point functions with zero external momenta became UV-finite, and so did the 2-loop integrals for vanishing external momenta. The basic reason is simple; even for the 1-loop diagrams, the suppression coming from the propagators is stronger than the enhancements coming from the vertices. This ensures two things - first, it makes the loops finite and, second, the UV growth of the finite diagrams with respect to the external momenta becomes weaker in every subsequent loops. Thus, finiteness of higher loops is ensured recursively.

With this adequate information, we now concentrate on the scattering problem for BGKM gravity. We can compute the $s, t, u$-channels, tree-level scattering diagram $p_{1} p_{2} \rightarrow p_{3} p_{4}$, see Fig. 1, which is given by in the Euclidean space, as:

$$
i \mathcal{T}_{\text {tree-level }}^{\text {s-chanel }}=\frac{1}{M_{P}^{2}} V\left(p_{1}, p_{2},-p_{1}-p_{2}\right) V\left(-p_{3},-p_{4}, p_{1}+p_{2}\right)\left(\frac{i}{s e^{-s / M^{2}}}\right) .
$$




$$
\begin{aligned}
i \mathcal{T}_{\text {tree-level }}^{\mathrm{t}-\text { chanel }} & =\frac{1}{M_{P}^{2}} V\left(p_{1},-p_{3}, p_{3}-p_{1}\right) V\left(p_{2},-p_{4}, p_{4}-p_{2}\right)\left(\frac{i}{t e^{-t / M^{2}}}\right), \\
i \mathcal{T}_{\text {tree-level }}^{\mathrm{u}-\text { channel }} & =\frac{1}{M_{P}^{2}} V\left(p_{1},-p_{4}, p_{4}-p_{1}\right) V\left(p_{2},-p_{3}, p_{3}-p_{2}\right)\left(\frac{i}{u e^{-u / M^{2}}}\right) .
\end{aligned}
$$

Therefore, we have

$$
\begin{aligned}
\mathcal{T}_{\text {tree-level }} & =\frac{1}{16 M_{P}^{2}\left(p_{1}+p_{2}\right)^{2} e^{\left(\bar{p}_{1}+\bar{p}_{2}\right)^{2}}}\left[p_{1}^{2}+p_{2}^{2}+\left(p_{1}+p_{2}\right)^{2}\right]\left[p_{3}^{2}+p_{4}^{2}+\left(p_{1}+p_{2}\right)^{2}\right] \\
& \times\left[1-e^{\bar{p}_{1}^{2}}-e^{\bar{p}_{2}^{2}}-e^{\left(\bar{p}_{1}+\bar{p}_{2}\right)^{2}}\right]\left[1-e^{\bar{p}_{3}^{2}}-e^{\bar{p}_{4}^{2}}-e^{\left(\bar{p}_{1}+\bar{p}_{2}\right)^{2}}\right] \\
& +\left(p_{2} \leftrightarrow-p_{3}\right) \\
& +\left(p_{2} \leftrightarrow-p_{4}\right)
\end{aligned}
$$

In the CM frame, we obtain:

$$
\begin{aligned}
\mathcal{T}_{\text {tree-level }} & =-\frac{1}{16 M_{P}^{2} s e^{-\frac{s}{M^{2}}}}(-2 s)^{2}\left(1-2 e^{-\frac{s}{2 M^{2}}}-e^{-\frac{s}{M^{2}}}\right)^{2} \\
& -\frac{1}{16 M_{P}^{2} t e^{-\frac{t}{M^{2}}}}(-s-t)^{2}\left(1-2 e^{-\frac{s}{2 M^{2}}}-e^{-\frac{t}{M^{2}}}\right)^{2} \\
& -\frac{1}{16 M_{P}^{2} u e^{-\frac{u}{M^{2}}}}(-s-u)^{2}\left(1-2 e^{-\frac{s}{2 M^{2}}}-e^{-\frac{u}{M^{2}}}\right)^{2} .
\end{aligned}
$$

Let us again point out that $s, t, u$ are all negative in Euclidean space and satisfy $s=u+t$. Clearly, the cross section $\sigma_{\text {tree-level }}$ corresponding to $\mathcal{T}_{\text {tree-level }}$ blows up as $s \rightarrow-\infty$ since $|\mathcal{T}|^{2}$ diverges in that limit.

Before we compute the scattering amplitude, let us first consider the 1-loop, 2point function, see Fig. 3, with arbitrary external momenta, which is given by

$$
\Gamma_{2,1}\left(p^{2}\right)=\frac{i}{2 i^{2} M_{P}^{2}} \int \frac{d^{4} k}{(2 \pi)^{4}} \frac{V^{2}\left(-p, \frac{p}{2}+k, \frac{p}{2}-k\right)}{\left(\frac{p}{2}+k\right)^{2}\left(\frac{p}{2}-k\right)^{2} e^{\left(\frac{\bar{p}}{2}+\bar{k}\right)^{2}} e^{\left(\frac{\bar{p}}{2}-\bar{k}\right)^{2}}} .
$$

Using the dimensional regularisation scheme, we obtain an $\epsilon^{-1}$ pole,

$$
\Gamma_{2,1, \mathrm{div}}\left(p^{2}\right)=\frac{i p^{4}}{64 \pi^{2} M_{P}^{2}} \frac{1}{\epsilon},
$$

as expected, which can be eliminated using a suitable counter-term. The counterterm, which is needed to cancel the $\epsilon^{-1}$ divergence and which should be added to the action in Eq. (4.12), is given by

$$
S_{\mathrm{ct}}=-\frac{1}{128 \epsilon \pi^{2} M_{P}^{2}} \int d^{4} x \phi \square^{2} \phi,
$$

yielding

$$
\Gamma_{2,1, \mathrm{ct}}\left(p^{2}\right)=-\frac{i p^{4}}{64 \pi^{2} M_{P}^{2}} \frac{1}{\epsilon}
$$


Had we employed a hard cut-off $\Lambda$, the maximum divergence would have been $\Lambda^{4}$.

Therefore, regarding the renormalised 1-loop, 2-point function, $\Gamma_{2,1 \mathrm{r}}$, with external momenta $p,-p$, we have $\Gamma_{2,1 \mathrm{r}}=\frac{i M^{4}}{M_{P}^{2}} f\left(\bar{p}^{2}\right)$, where

$$
\begin{aligned}
f\left(\bar{p}^{2}\right) & =\frac{\bar{p}^{4}}{128 \pi^{2}}\left(-\log \left(\frac{\bar{p}^{2}}{4 \pi}\right)-\gamma+2\right) \\
& +\frac{e^{-\bar{p}^{2}}}{512 \pi^{2} \bar{p}^{2}}\left[-2 e^{\bar{p}^{2}}\left(e^{2 \bar{p}^{2}}-1\right) \bar{p}^{6} E i\left(-\bar{p}^{2}\right)+\left(e^{\bar{p}^{2}}-1\right)\left(-2\left(\bar{p}^{4}+3 \bar{p}^{2}+2\right)\right.\right. \\
& \left.\left.+\left(e^{\frac{3 \bar{p}^{2}}{2}}-e^{\frac{\bar{p}^{2}}{2}}\right)\left(2 \bar{p}^{4}+5 \bar{p}^{2}+4\right)+e^{\bar{p}^{2}}\left(e^{\bar{p}^{2}}-1\right) \bar{p}^{6} E i\left(-\frac{\bar{p}^{2}}{2}\right)+2 e^{\bar{p}^{2}}\left(7\left(\bar{p}^{4}+\bar{p}^{2}\right)+2\right)\right)\right] .
\end{aligned}
$$

Now, regarding the 1-loop scattering diagram, see Fig. 4, we obtain:

$$
\begin{aligned}
\mathcal{T}_{1-\text { loop }} & =V\left(p_{1}, p_{2},-p_{1}-p_{2}\right) V\left(-p_{3},-p_{4}, p_{1}+p_{2}\right)\left(\frac{i}{s e^{-s / M^{2}}}\right)^{2} \frac{M^{4}}{M_{P}^{4}} f(-s) \\
& +V\left(p_{1},-p_{3}, p_{3}-p_{1}\right) V\left(p_{2},-p_{4}, p_{1}-p_{3}\right)\left(\frac{i}{t e^{-t / M^{2}}}\right)^{2} \frac{M^{4}}{M_{P}^{4}} f(-t) \\
& +V\left(p_{1},-p_{4}, p_{4}-p_{1}\right) V\left(p_{2},-p_{3}, p_{1}-p_{4}\right)\left(\frac{i}{u e^{-u / M^{2}}}\right)^{2} \frac{M^{4}}{M_{P}^{4}} f(-u)
\end{aligned}
$$

where $\Gamma_{2,1 \mathrm{r}}=\frac{i M^{4}}{M_{P}^{2}} f(-s)=\frac{i M^{4}}{M_{P}^{2}} f\left(\bar{p}^{2}\right)$, where $f\left(\bar{p}^{2}\right)$ is given by Eq. 4.30) and $f\left(\bar{p}^{2}\right)$ is a regular analytic function of $\bar{p}^{2}$ which grows as $e^{\frac{3 \bar{p}^{2}}{2}}$ as $\bar{p}^{2} \rightarrow \infty$.

As $s \rightarrow-\infty, \Gamma_{2,1 \mathrm{r}}(-s)$ (and $\left.f(-s)\right)$ goes as $e^{-\frac{3 s}{2 M^{2}}}$. The $s$-channel of $\mathcal{T}_{1-\text { loop }}$ goes as $e^{-\frac{2 s}{M^{2}}} e^{\frac{2 s}{M^{2}}} e^{-\frac{3 s}{2 M^{2}}}=e^{-\frac{3 s}{2 M^{2}}}$ when $s \rightarrow-\infty$. As $s \rightarrow-\infty, \mathcal{T}_{1-\text { loop }}^{\text {s-chanel }}$ diverges. $\mathcal{T}_{1-\text { loop }}^{\mathrm{t} \text {-channel }}$ and $\mathcal{T}_{1-\text { loop }}^{\mathrm{u} \text {-channel }}$ also diverge.

\subsection{Dressing the propagator and the vertices}

Similar to the earlier cases, we have found that dressed propagator is more strongly exponentially suppressed than the bare propagator. Since $\Pi\left(p^{2}\right) \Gamma_{2,1 \mathrm{r}}\left(p^{2}\right)$ grows with large momenta, we have, for large $p$,

$$
\widetilde{\Pi}\left(p^{2}\right) \rightarrow \Gamma_{2,1 \mathrm{r}}^{-1}\left(p^{2}\right) \approx\left(9-12 \bar{p}^{-2}\right)^{-1} e^{-\frac{3 \bar{p}^{2}}{2}} .
$$


Now, if we replace the bare propagator with the dressed propagator in the tree-level scattering diagrams, see Fig. 5 (bottom), we obtain:

$$
\begin{aligned}
\mathcal{T}_{\text {dressed }} & =V\left(p_{1}, p_{2},-p_{1}-p_{2}\right) V\left(-p_{3},-p_{4}, p_{1}+p_{2}\right)\left(\frac{1}{M_{P}^{2} s e^{-s / M^{2}}+M^{4} f(-s)}\right) \\
& +V\left(p_{1},-p_{3}, p_{3}-p_{1}\right) V\left(p_{2},-p_{4}, p_{1}-p_{3}\right)\left(\frac{1}{M_{P}^{2} t e^{-t / M^{2}}+M^{4} f(-t)}\right) \\
& +V\left(p_{1},-p_{4}, p_{4}-p_{1}\right) V\left(p_{2},-p_{3}, p_{1}-p_{4}\right)\left(\frac{1}{M_{P}^{2} u e^{-u / M^{2}}+M^{4} f(-u)}\right),
\end{aligned}
$$

where, as $s \rightarrow-\infty, f(-s)$ goes as $e^{-\frac{3 s}{2 M^{2}}}$. An explicit computation, see Fig. 5 (bottom), gives us, as $s \rightarrow-\infty$,

$$
\begin{aligned}
& \mathcal{T}_{\text {dressed }}^{\text {s-channel }} \sim\left[2 e^{-\frac{s}{2 M^{2}}}+e^{-\frac{s}{M^{2}}}-1\right]^{2} e^{\frac{3 s}{2 M^{2}}} \sim e^{-\frac{s}{2 M^{2}}} \\
& \mathcal{T}_{\text {dressed }}^{\mathrm{t}-\text { channel }} \sim\left[2 e^{-\frac{s}{2 M^{2}}}+e^{-\frac{t}{M^{2}}}-1\right]^{2} e^{\frac{3 t}{2 M^{2}}}=\left[2 e^{-\frac{s}{2 M^{2}}}+e^{-\frac{s(1-\cos \theta)}{2 M^{2}}}-1\right]^{2} e^{\frac{3 s(1-\cos \theta)}{4 M^{2}}} \\
& \mathcal{T}_{\text {dressed }}^{\mathrm{u}-\text { channel }} \sim\left[2 e^{-\frac{s}{2 M^{2}}}+e^{-\frac{u}{M^{2}}}-1\right]^{2} e^{\frac{3 u}{2 M^{2}}}=\left[2 e^{-\frac{s}{2 M^{2}}}+e^{-\frac{s(1+\cos \theta)}{2 M^{2}}}-1\right]^{2} e^{\frac{3 s(1+\cos \theta)}{4 M^{2}}}
\end{aligned}
$$

Hence, we can make the following observations:

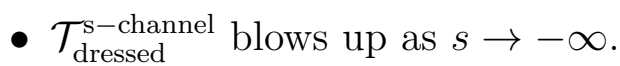

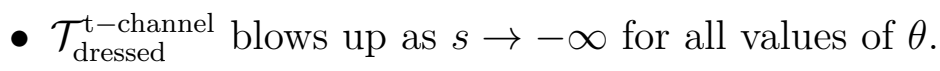

- $\mathcal{T}_{\text {dressed }}^{\mathrm{u}-\text { channel }}$ blows up as $s \rightarrow-\infty$ for all values of $\theta$.

Since $\mathcal{T}_{\text {dressed }}=\mathcal{T}_{\text {dressed }}^{\text {s-channel }}+\mathcal{T}_{\text {dressed }}^{\mathrm{t} \text {-chanel }}+\mathcal{T}_{\text {dressed }}^{\mathrm{u} \text {-channel }}$, one can verify that the total cross section $\sigma_{\text {dressed }}$ corresponding to $\mathcal{T}_{\text {dressed }}$ blows up as $s \rightarrow-\infty$. We also observe that the external momentum dependence of $\mathcal{T}_{\text {dressed }}$ grows less for large external momenta as compared to the external momentum dependence of $\mathcal{T}_{\text {tree-level }}$ (or $\mathcal{T}_{1 \text {-loop }}$ ). Hence, the use of the dressed propagator ameliorates the external momentum growth of the scattering diagrams, but it is not sufficient by itself.

To see whether we can eliminate the external momentum growth of the scattering diagrams, we will dress the vertices by making renormalised vertex loop corrections to the bare vertices at the left- and right-ends of the scattering diagrams, see Fig. 7. Following exactly the same prescription as in section 3.2 , we obtain the relation

$$
\alpha^{n}=\beta^{n}=\gamma^{n}=\alpha^{n-1}+\frac{1}{3}\left(\beta^{n-1}+\gamma^{n-1}\right)-\frac{1}{3},
$$

which is Eq. (3.23). Since $\alpha^{0}=1$ and $\beta^{0}=\gamma^{0}=0$, we observe that the coefficients $\alpha^{n}, \beta^{n}$ and $\gamma^{n}$ increase as $n$ increases; thus, dressing the vertices by keeping the 
propagators bare and making just vertex loop corrections to the bare vertices at the left- and right-ends of the scattering diagrams cannot tame the external momentum growth of the scattering diagrams.

For that reason, and as an example, we will now dress the bare vertices at the leftand right-ends of the scattering diagram whose scattering matrix element is $\mathcal{T}_{\text {dressed }}$ by making both propagator and vertex loop corrections to the said vertices, see Fig. 8 . Following the same reasoning as in section $3.3, \alpha^{n}, \beta^{n}$ and $\gamma^{n}$ become negative for $n \geq 4$.

For $n=4$, we have the following conclusions:

- As in section 3.3, the largest external momentum contribution of the $s$-channel, see Fig. 9 , goes as

$$
e^{\frac{44 s}{27 M^{2}}} e^{\frac{3 s}{2 M^{2}}}=e^{\frac{169 s}{54 M^{2}}}
$$

which tends to 0 as $s \rightarrow-\infty$.

- The largest external momentum contribution of the $t$-channel goes as

$$
e^{\frac{22 t}{27 M^{2}}} e^{\frac{22 s}{27 M^{2}}} e^{\frac{3 t}{2 M^{2}}}=e^{\frac{s(213-125 \cos \theta)}{108 M^{2}}}
$$

which, again, tends to 0 as $s \rightarrow-\infty$ for all values of $\theta$.

- The largest external momentum contribution of the $u$-channel goes as

$$
e^{\frac{22 u}{27 M^{2}}} e^{\frac{22 s}{27 M^{2}}} e^{\frac{3 u}{2 M^{2}}}=e^{\frac{s(213+125 \cos \theta)}{108 M^{2}}},
$$

which, again, tends to 0 as $s \rightarrow-\infty$ for all values of $\theta$. Hence, for sufficiently large $n$ (specifically, for $n \geq 4$ ), there is no exponential growth for the $s^{-}, t$ - and $u$-channels as $s \rightarrow-\infty$. The external momentum growth of $\mathcal{T}_{\text {tree-level }}, \mathcal{T}_{1-\text { loop }}$ etc. would also be eliminated following this prescription at sufficiently high loop order.

We observe that, for sufficiently large $n$, dressing the vertices by making both propagator and vertex loop corrections to the bare vertices at the left- and rightends of the scattering diagrams makes the external momentum dependence of any scattering diagram convergent in the UV. By considering renormalised propagator and vertex loop corrections to the bare vertices, we can eliminate the external momentum growth appearing in scattering diagrams in the regime of large external momenta, i.e., as $s \rightarrow-\infty$. In contrast, dressing the vertices by considering just vertex loop corrections to the bare vertices is not sufficient. Thus, dressing the vertices by making both propagator and vertex loop corrections to the bare vertices is essential to taming the external momentum growth of scattering diagrams in the UV and, as a result, we expect the cross sections of those diagrams to be finite (see Eq. A.4 in appendix A for the relation between the differential cross section $d \sigma$ and the scattering matrix element $\mathcal{T}$ ). 


\section{Conclusions}

The aim of this paper has been to examine the external momentum dependence of scattering diagrams in the context of infinite-derivative field theories and gravity. We have found that for a finite-order, higher-derivative scalar field theory the cross section of tree-level scattering diagrams blows up at large momenta. Even considering dressed propagators and dressed vertices, by making propagator and vertex loop corrections to the bare vertices of the scattering diagrams, is not sufficient to eliminate the external momentum growth. However, we have noticed that dressing the propagators indeed ameliorates the external momentum growth a bit. Motivated by these results, we studied an infinite-derivative, non-local scalar field theory with non-local interactions. In this setup, the propagators are exponentially suppressed and the vertices are exponentially enhanced.

For such non-local interactions, we have found that the tree-level cross section still blows up in the UV. Also, dressing the propagator is not sufficient to tame the growth. On the other hand, dressing the bare vertices by making renormalised propagator and vertex loop corrections to the bare vertices at sufficiently high loop order (when the loop order $n$ satisfies $n \geq 4$ ) can potentially yield finiteness of the cross section in the UV. What leads to this conclusion is the softening of the vertices. At higher loop order, the dressed vertices lead to negative exponents, which effectively softens any high-energy scattering amplitude. As a result, the scattering cross section is expected not to blow up for large external momenta, which is encouraging as to the infinite-derivative theories of gravity under consideration. We may speculate that, for such cases, scattering scalar wave packets with non-local interactions would not lead to black hole singularity. This is indeed an interesting result which can help us to understand the UV properties of gravity, if gravity itself were treated non-locally in the UV.

This motivates us to study high-energy scattering diagrams in a scalar toy-model inspired by the non-local, singularity-free theory of gravity introduced in Ref. [59]. In this case, we were able to demonstrate that dressing the vertices and the propagators indeed leads to a cross section that is expected to be finite for the scattering diagrams, which become convergent in the ultraviolet. This gives rise to a very interesting possibility that perhaps our recipe could be followed for pure gravity, as in the case of BGKM, to show that such non-locality indeed softens the trans-Planckian scattering problem and can avoid forming a black hole singularity. We believe that our results will have consequences for understanding problems such as black hole singularity and the cosmological singularity problem in a time-dependent setup. 


\section{Acknowledgements}

The authors would like to thank Tirthabir Biswas, Aindriú Conroy, Valery Frolov, Steven Giddings, Alex Koshelev, Warren Siegel, Ali Teimouri, Terry Tomboulis for helpful discussions. ST is supported by a scholarship from the Onassis Foundation and AM is supported by the STFC grant ST/J000418/1.

\section{Appendix}

\section{A Definitions and Conventions in Euclidean Space}

Let us define $s=-\left(p_{1}+p_{2}\right)^{2}=-\left(p_{3}+p_{4}\right)^{2}=-E_{\mathrm{CM}}^{2}$, where $p_{1}+p_{2}=p_{3}+p_{4}$. Moreover, $t=-\left(p_{1}-p_{3}\right)^{2}=-\left(p_{2}-p_{4}\right)^{2}$ and $u=-\left(p_{1}-p_{4}\right)^{2}=-\left(p_{2}-p_{3}\right)^{2}$. We have that $s, t, u$ are all negative in Euclidean space and satisfy $s=u+t$. We should keep in mind that we consider massless particles in this paper and, in Minkowski space ("mostly plus" metric signature), $p_{i}^{2}=-m_{i}^{2}=0$, where $i=1,2,3,4$.

The total cross section, $\sigma$, in the centre-of-mass (CM) frame is given by

$$
\sigma=\frac{1}{S} \int_{t_{\min }}^{t_{\max }} d t \frac{d \sigma}{d t},
$$

where $t_{\min }$ and $t_{\max }$ are given by

$$
t=-2 E_{1} E_{3}+2\left|\mathbf{p}_{1}\right|\left|\mathbf{p}_{3}\right| \cos \theta,
$$

with $\cos \theta=-1$ and +1 , respectively ( $\theta$ is the angle between $\left|\mathbf{p}_{1}\right|$ and $\left|\mathbf{p}_{3}\right|$ ). $S$ is the symmetry factor for $n_{i}^{\prime}$ identical outgoing particles of type $i$,

$$
S=\prod_{i} n_{i}^{\prime} !
$$

and, for two outgoing particles (after we analytically continue to Euclidean space), we have

$$
\frac{d \sigma}{d t}=-\frac{1}{64 \pi s\left|\mathbf{p}_{1}\right|^{2}}|\mathcal{T}|^{2}
$$

where $\mathcal{T}$ is the scattering matrix element. In the CM frame, we also have

$$
\left|\mathbf{p}_{1}\right|=\left|\mathbf{p}_{2}\right|=\left|\mathbf{p}_{3}\right|=\left|\mathbf{p}_{4}\right|=E_{1}=E_{2}=E_{3}=E_{4}=\frac{\sqrt{-s}}{2}
$$

Furthermore, we have that $t_{\min }=s$ and $t_{\max }=0$. Since the two outgoing particles are identical, the symmetry factor is $S=2$. Moreover, in Euclidean space,

$$
t=\frac{s}{2}(1-\cos \theta)
$$

and

$$
u=\frac{s}{2}(1+\cos \theta) \text {. }
$$




\section{References}

[1] S. Weinberg, "The Quantum theory of fields. Vol. 1: Foundations,"

[2] H. Elvang and Y. t. Huang, "Scattering Amplitudes," arXiv:1308.1697 [hep-th].

[3] D. Amati, M. Ciafaloni and G. Veneziano, "Planckian scattering beyond the semiclassical approximation," Phys. Lett. B 289, 87 (1992). doi:10.1016/03702693(92)91366-H

[4] M. Fabbrichesi, R. Pettorino, G. Veneziano and G. A. Vilkovisky, "Planckian energy scattering and surface terms in the gravitational action," Nucl. Phys. B 419, 147 (1994) [hep-th/9309037].

[5] G. Veneziano and J. Wosiek, "Exploring an S-matrix for gravitational collapse," JHEP 0809, 023 (2008) [arXiv:0804.3321 [hep-th]].

[6] D. Amati, M. Ciafaloni and G. Veneziano, "Towards an S-matrix description of gravitational collapse," JHEP 0802, 049 (2008) [arXiv:0712.1209 [hep-th]].

[7] G. Veneziano, "String-theoretic unitary S-matrix at the threshold of black-hole production," JHEP 0411, 001 (2004) [hep-th/0410166].

[8] S. B. Giddings, M. Schmidt-Sommerfeld and J. R. Andersen, "High energy scattering in gravity and supergravity," Phys. Rev. D 82, 104022 (2010) [arXiv:1005.5408 [hep-th]].

[9] S. B. Giddings and M. Srednicki, "High-energy gravitational scattering and black hole resonances," Phys. Rev. D 77, 085025 (2008) [arXiv:0711.5012 [hep-th]].

[10] S. B. Giddings and S. D. Thomas, "High-energy colliders as black hole factories: The End of short distance physics," Phys. Rev. D 65, 056010 (2002) [hep-ph/0106219].

[11] D. M. Eardley and S. B. Giddings, "Classical black hole production in highenergy collisions," Phys. Rev. D 66, 044011 (2002) [gr-qc/0201034].

[12] J. Polchinski, "String theory. Vols. 1, and 2," Cambridge University Press (1998).

[13] G. Veneziano, "Construction of a crossing - symmetric, Regge behaved amplitude for linearly rising trajectories," Nuovo Cim. A 57, 190 (1968).

[14] D. J. Gross and P. F. Mende, "String Theory Beyond the Planck Scale," Nucl. Phys. B 303, 407 (1988).

[15] S. B. Giddings, D. J. Gross and A. Maharana, "Gravitational effects in ultrahighenergy string scattering," Phys. Rev. D 77, 046001 (2008) [arXiv:0705.1816 [hepth]]. 
[16] D. J. Gross and P. F. Mende, "The High-Energy Behavior of String Scattering Amplitudes," Phys. Lett. B 197, 129 (1987).

[17] W. Staessens and B. Vercnocke, "Lectures on Scattering Amplitudes in String Theory," arXiv:1011.0456 [hep-th].

[18] A. Ashtekar, "Introduction to loop quantum gravity and cosmology," Lect. Notes Phys. 863, 31 (2013).

[19] for a review, see: H. Nicolai, K. Peeters and M. Zamaklar, "Loop quantum gravity: An Outside view," Class. Quant. Grav. 22, R193 (2005) [hep-th/0501114].

[20] for a review, see: J. Henson, "The Causal set approach to quantum gravity," In *Oriti, D. (ed.): Approaches to quantum gravity* 393-413 [gr-qc/0601121].

[21] P. Donà, S. Giaccari, L. Modesto, L. Rachwal and Y. Zhu, "Scattering amplitudes in super-renormalizable gravity," JHEP 1508, 038 (2015) [arXiv:1506.04589 [hep-th]].

[22] D. Amati, M. Ciafaloni and G. Veneziano, "Planckian scattering beyond the semiclassical approximation," Phys. Lett. B 289, 87 (1992).

[23] D. Amati, M. Ciafaloni and G. Veneziano, "Effective action and all order gravitational eikonal at Planckian energies," Nucl. Phys. B 403, 707 (1993).

[24] D. Amati, M. Ciafaloni and G. Veneziano, "Higher Order Gravitational Deflection and Soft Bremsstrahlung in Planckian Energy Superstring Collisions," Nucl. Phys. B 347, 550 (1990).

[25] D. Amati, M. Ciafaloni and G. Veneziano, "Can Space-Time Be Probed Below the String Size?," Phys. Lett. B 216, 41 (1989).

[26] D. Amati, M. Ciafaloni and G. Veneziano, "Superstring Collisions at Planckian Energies," Phys. Lett. B 197, 81 (1987).

[27] A. A. Tseytlin, "On singularities of spherically symmetric backgrounds in string theory," Phys. Lett. B 363, 223 (1995) [hep-th/9509050].

[28] W. Siegel, "Stringy gravity at short distances," hep-th/0309093.

[29] T. Biswas, M. Grisaru and W. Siegel, "Linear Regge trajectories from worldsheet lattice parton field theory," Nucl. Phys. B 708, 317 (2005) [hep-th/0409089].

[30] T. Biswas and N. Okada, "Towards LHC physics with nonlocal Standard Model," Nucl. Phys. B 898, 113 (2015) [arXiv:1407.3331 [hep-ph]].

[31] T. Biswas, J. A. R. Cembranos and J. I. Kapusta, "Thermal Duality and Hagedorn Transition from p-adic Strings," Phys. Rev. Lett. 104, 021601 (2010) [arXiv:0910.2274 [hep-th]]. 
[32] T. Biswas, J. A. R. Cembranos and J. I. Kapusta, "Thermodynamics and Cosmological Constant of Non-Local Field Theories from p-Adic Strings," JHEP 1010, 048 (2010) [arXiv:1005.0430 [hep-th]].

[33] T. Biswas, J. A. R. Cembranos and J. I. Kapusta, "Finite Temperature Solitons in Non-Local Field Theories from p-Adic Strings," Phys. Rev. D 82 (2010) 085028 [arXiv:1006.4098 [hep-th]].

[34] T. Biswas, J. Kapusta and A. Reddy, "Thermodynamics of String Field Theory Motivated Nonlocal Models," JHEP 1212, 008 (2012) [arXiv:1201.1580 [hep-th]].

[35] E. Tomboulis, "Renormalizability and Asymptotic Freedom in Quantum Gravity," Phys. Lett. B 97, 77 (1980).

E. T. Tomboulis, "Renormalization And Asymptotic Freedom In Quantum Gravity," In *Christensen, S.m. ( Ed.): Quantum Theory Of Gravity*, 251-266 and Preprint - TOMBOULIS, E.T. (REC.MAR.83) 27p.

E. T. Tomboulis, "Superrenormalizable gauge and gravitational theories," hepth/9702146.

E. T. Tomboulis, "Non-local and quasi-local field theories," arXiv:1507.00981 [hep-th].

[36] A. Borde, A. H. Guth and A. Vilenkin, "Inflationary space-times are incompletein past directions," Phys. Rev. Lett. 90, 151301 (2003) [gr-qc/0110012].

[37] T. Biswas, A. Mazumdar and W. Siegel, "Bouncing universes in string-inspired gravity," JCAP 0603, 009 (2006) [hep-th/0508194].

[38] T. Biswas, E. Gerwick, T. Koivisto and A. Mazumdar, "Towards singularity and ghost free theories of gravity," Phys. Rev. Lett. 108, 031101 (2012) [arXiv:1110.5249 [gr-qc]].

[39] T. Biswas, T. Koivisto and A. Mazumdar, "Nonlocal theories of gravity: the flat space propagator," arXiv:1302.0532 [gr-qc].

[40] L. Modesto, "Super-renormalizable Quantum Gravity," Phys. Rev. D 86, 044005 (2012) [arXiv:1107.2403 [hep-th]].

[41] N. Barnaby and N. Kamran, "Dynamics with infinitely many derivatives: The Initial value problem," JHEP 0802, 008 (2008) [arXiv:0709.3968 [hep-th]].

N. Barnaby, "A New Formulation of the Initial Value Problem for Nonlocal Theories," Nucl. Phys. B 845, 1 (2011) [arXiv:1005.2945 [hep-th]].

[42] E. Witten, "Noncommutative Geometry and String Field Theory," Nucl. Phys. $B$ 268, 253 (1986).

[43] P. G. O. Freund and M. Olson, "Nonarchimedean Strings," Phys. Lett. B 199, 186 (1987).

P. G. O. Freund and E. Witten, "Adelic String Amplitudes," Phys. Lett. B 199, 
191 (1987).

L. Brekke, P. G. O. Freund, M. Olson and E. Witten, "Nonarchimedean String Dynamics," Nucl. Phys. B 302, 365 (1988).

P. H. Frampton and Y. Okada, "Effective Scalar Field Theory of $P^{-}$adic String," Phys. Rev. D 37, 3077 (1988).

[44] D. J. Gross and A. Jevicki, "Operator Formulation of Interacting String Field Theory," Nucl. Phys. B 283, 1 (1987).

D. J. Gross and A. Jevicki, "Operator Formulation of Interacting String Field Theory. 2.," Nucl. Phys. B 287, 225 (1987).

[45] T. Biswas, A. S. Koshelev and A. Mazumdar, "Gravitational theories with stable (anti-)de Sitter backgrounds," arXiv:1602.08475 [hep-th].

[46] G. V. Efimov, "Non-Local Quantum Theory of the Scalar Field," Commun. Math. Phys. 5, 42 (1967).

G. V. Efimov and S. Z. Seltser, "Gauge invariant non-local theory of the weak interactions," Annals Phys. 67, 124 (1971).

G. V. Efimov, "On the construction of non-local quantum electrodynamics," Annals Phys. 71, 466 (1972).

V. A. Alebastrov and G. V. Efimov, "A proof of the unitarity of S matrix in a non-local quantum field theory," Commun. Math. Phys. 31, 1 (1973).

V. A. Alebastrov and G. V. Efimov, "Causality In The Quantum Field Theory With The non-local Interaction," Commun. Math. Phys. 38, 11 (1974).

[47] T. Biswas, T. Koivisto and A. Mazumdar, "Towards a resolution of the cosmological singularity in non-local higher derivative theories of gravity," JCAP 1011, 008 (2010) [arXiv:1005.0590 [hep-th]].

[48] T. Biswas, A. S. Koshelev, A. Mazumdar and S. Y. Vernov, "Stable bounce and inflation in non-local higher derivative cosmology," JCAP 1208, 024 (2012) [arXiv:1206.6374 [astro-ph.CO]].

[49] A. Conroy, A. S. Koshelev and A. Mazumdar, "Geodesic completeness and homogeneity condition for cosmic inflation," Phys. Rev. D 90, no. 12, 123525 (2014) [arXiv:1408.6205 [gr-qc]].

[50] D. Chialva and A. Mazumdar, "Cosmological implications of quantum corrections and higher-derivative extension," Mod. Phys. Lett. A 30, no. 03n04, 1540008 (2015) [arXiv:1405.0513 [hep-th]].

[51] B. Craps, T. De Jonckheere and A. S. Koshelev, "Cosmological perturbations in non-local higher-derivative gravity," JCAP 1411, no. 11, 022 (2014) [arXiv:1407.4982 [hep-th]].

[52] T. Biswas, A. Conroy, A. S. Koshelev and A. Mazumdar, "Generalized ghostfree quadratic curvature gravity," Class. Quant. Grav. 31, 015022 (2014) [Class. Quant. Grav. 31, 159501 (2014)] [arXiv:1308.2319 [hep-th]]. 
[53] A. Conroy, A. Mazumdar, S. Talaganis and A. Teimouri, "Nonlocal gravity in D dimensions: Propagators, entropy, and a bouncing cosmology," Phys. Rev. D 92, no. 12, 124051 (2015) [arXiv:1509.01247 [hep-th]].

[54] V. P. Frolov and A. Zelnikov, "Head-on collision of ultra-relativistic particles in ghost-free theories of gravity," arXiv:1509.03336 [hep-th].

[55] V. P. Frolov, "Mass-gap for black hole formation in higher derivative and ghost free gravity," Phys. Rev. Lett. 115, no. 5, 051102 (2015) [arXiv:1505.00492 [hepth]].

[56] V. P. Frolov, A. Zelnikov and T. de Paula Netto, "Spherical collapse of small masses in the ghost-free gravity," JHEP 1506, 107 (2015) [arXiv:1504.00412 [hep-th]].

[57] B. Holdom, "On the fate of singularities and horizons in higher derivative gravity," Phys. Rev. D 66, 084010 (2002) [hep-th/0206219].

[58] H. Lü, A. Perkins, C. N. Pope and K. S. Stelle, "Spherically Symmetric Solutions in Higher-Derivative Gravity," Phys. Rev. D 92, no. 12, 124019 (2015) [arXiv:1508.00010 [hep-th]].

[59] S. Talaganis, T. Biswas and A. Mazumdar, "Towards understanding the ultraviolet behavior of quantum loops in infinite-derivative theories of gravity," Class. Quant. Grav. 32, no. 21, 215017 (2015) [arXiv:1412.3467 [hep-th]].

[60] P. Van Nieuwenhuizen, "On ghost-free tensor lagrangians and linearized gravitation," Nucl. Phys. B 60, 478 (1973). 